\title{
NONPOSITIVE CURVATURE AND REFLECTION GROUPS
}

\author{
Michael W. Davis $(*)$
}

\section{Introduction.}

A space is aspherical if its universal cover is contractible. Examples of aspherical spaces occur in differential geometry (as complete Riemannian manifolds of nonpositive sectional curvature), in Lie groups (as $\Gamma \backslash G / K$ where $G$ is a Lie group, $K$ is a maximal compact subgroup and $\Gamma$ is a discrete torsion-free subgroup), in 3 -manifold theory and as certain 2-dimensional cell complexes. The main purpose of this paper is to describe another interesting class of examples coming from the theory of reflection groups (also called "Coxeter groups"). One of the main results is explained in $\S 3$ and $\S 9$ : given a finite simplicial complex $L$, there is a compact aspherical polyhedron $X$ such that the link of each vertex in $X$ is isomorphic to the barycentric subdivision of $L$. A version of this result first appeared in [D1]. Later, in $[\mathrm{G}]$, Gromov showed that the polyhedron $X$ can be given a piecewise Euclidean metric which is nonpositively curved in the sense of Aleksandrov. This gives a new proof of the asphericity of $X$ (cf. Theorem 1.5 below). As we vary the choice of the link $L$ we get polyhedra $X$ with a variety of interesting properties. For example, if $L$ is homeomorphic to an $(n-1)$-sphere, then $X$ is an $n$-manifold. More examples are discussed in $\S 11$.

Chapter I covers background material on nonpositive curvature. The main examples are discussed in Chapter II. Chapter III deals with some related aspherical complexes which arise in the study of complements of arrangements of hyperplanes.

This paper began as a set of notes for three lectures which I gave at the Eleventh Annual Workshop in Geometric Topology at Park City, Utah in June 1994. In the course of preparing it for this volume I have added approximately 25 percent more material, notably, $\S 4, \S 5, \S 10$ and parts of $\S 11$. I would like to thank Lonette Stoddard for preparing the figures. 


\section{CONTENTS}

Chapter I:Nonpositively Curved Spaces

$\S 1$. The $C A T(\epsilon)$-inequality

$\S 2$. Piecewise constant curvature polyhedra

$\S 3$. The $C A T(1)$ condition for links

a) Gromov's Lemma

b) Moussong's Lemma

c) Orthogonal joins

d) Spherical buildings

e) Polar duals of hyperbolic cells

f) Branched covers of round spheres

$\S 4$. Infinitesimal shadows and the ideal boundary

$\S 5$. Some well-known results in geometric topology and

their implications for CAT (0) spaces

$\S 6$. Euler characteristics and the Combinatorial Gauss-Bonnet Theorem

\section{Chapter II:Coxeter Groups}

$\S 7$. Coxeter systems

§. Coxeter cells

$\S 9$. The cell complex $\Sigma$ (in the case where $W$ is infinite)

$\S 10$. Reflection groups

§11. Applications and examples
a) Two dimensional complexes
b) Word hyperbolic Coxeter groups
c) Buildings
d) When is $\Sigma$ a manifold? A homology manifold?
e) Cohomological dimension
f) The Eilenberg-Ganea Problem
g) Is $\Sigma$ homeomorphic to $\mathbb{R}^{n}$ ?

\section{Chapter III:Artin Groups}

$\S 12$. Hyperplane complements

$\S 13$. The Salvetti complex

$\S 14$. Complexes of groups

$\S 15$. Reinterpretation of the Main Conjecture

$\S 16$. A cubical structure on $\Phi$

\section{References.}




\section{Nonpositively Curved Spaces.}

The notion of "nonpositive curvature" (or more generally of "curvature bounded above by a real number $\epsilon$ " makes sense for a more general class of metric spaces than Riemannian manifolds: one need only assume that any two points can be connected by a geodesic segment. For such spaces, the concept of curvature bounded above by $\epsilon$ can be defined via comparison triangles and the so-called "CAT( $\epsilon$-inequality". (This terminology is due to Gromov.) This is explained in $\S 1$. In $\S 2$ we consider "piecewise constant curvature polyhedra" and give a condition (in terms of links of vertices) for such a polyhedron to have curvature bounded from above. The condition is that each link be $C A T(1)$. In $\S 3$ we discuss criteria for such a link to be $C A T(1)$. The two conditions we are most interested in are given in Gromov's Lemma and Moussong's Lemma. These give criteria for piecewise spherical simplicial complexes (with sufficiently big simplices) to be $C A T(1)$. In $\S 4$ we show that a proper CAT (0) space can be compactified by adding an ideal boundary consisting of "endpoints" of geodesic rays. In this section we also discuss the notion of an "infinitesimal shadow" which measures the nonuniqueness of geodesic continuation at a point. In $\S 5$ we sketch a proof of Theorem 5.5 which asserts that the compactification of a CAT (0) PL manifold is homeomorphic to a disk. It is also indicated how such a result might fail in the non $P L$ context. (Explicit examples of this failure are given in $\S 11 \mathrm{~g}$ ).) In $\S 6$ we discuss a conjecture of H. Hopf concerning the Euler characteristic of a closed, nonpositively curved, even-dimensional manifold. Using the combinatorial version of the Gauss-Bonnet Theorem this leads us to a conjecture concerning a number associated to a piecewise spherical structure on an odd-dimensional sphere.

\section{$\S 1$. The $C A T(\epsilon)$-inequality.}

Given a smooth Riemannian manifold $M$ one defines its "curvature tensor" and from this its "sectional curvature". The sectional curvature $K$ of $M$ is a real-valued function on the set of all pairs $(x, P)$ where $x$ is a point in $M$ and $P$ is a tangent 2 -plane at $x$. Given a real number $\epsilon$, we say that " $M$ has curvature $\leq \epsilon$ " and write $K(M) \leq \epsilon$ if the sectional curvature $K$ is bounded above by $\epsilon$.

It has long been recognized that the condition that the curvature of $M$ is bounded above is equivalent to a condition which can be phrased purely in terms of the underlying metric (i.e., in terms of the distance function) on $M$. In fact, there are several possible versions of such a condition. We shall focus on one called the "CAT( $\epsilon)$ condition" by Gromov. ("C" stands for either "Cartan" or "comparison", " $A$ " for "Aleksandrov", and " $T$ " for "Toponogov".) Once one has such a condition one can define the notion of "curvature $\leq \epsilon$ " for many "singular" metric spaces, that is, for a more general class of metric spaces than Riemannian manifolds.

Good references for this material are $[\mathrm{GH}]$ and $[\mathrm{BH}]$.

We begin by stating the following Comparison Theorem of Aleksandrov. A proof can be found in the article of M. Troyanov in [GH].

Theorem 1.1. (Aleksandrov). Let $M$ be a simply connected, complete Riemannian manifold and $\epsilon$ a real number. Then $K(M) \leq \epsilon$ if and only if each geodesic triangle in $M$ (of perimeter $\leq 2 \pi / \sqrt{\epsilon}$ ) satisfies the $C A T(\epsilon)$-inequality.

A "geodesic triangle" in $M$ means a configuration in $M$ consisting of three points (the "vertices") and three (minimal) geodesic segments connecting them 
As $\epsilon>0,=0$, or $<0$, let $M_{\epsilon}^{2}$ stand for $S_{\epsilon}^{2}$ (the 2-sphere of constant curvature $\epsilon) \mathbb{E}^{2}$ (the Euclidean plane) or $\mathbb{H}_{\epsilon}^{2}$ (the hyperbolic plane of curvature $\epsilon$ ).

Let $T$ be a geodesic triangle in $M$. A comparison triangle for $T$ is a geodesic triangle $T^{*}$ in $M_{\epsilon}^{2}$ with the same edge lengths as $T$. Choose a vertex $x$ of $T$ and a point $y$ on the opposite edge. Let $x^{*}$ and $y^{*}$ denote the corresponding points in $T^{*}$. (See Figure 1).

The $C A T(\epsilon)$-inequality is

Figure 1

$$
d(x, y) \leq d^{*}\left(x^{*}, y^{*}\right)
$$

where $d$ and $d^{*}$ denote distance in $M$ and $M_{\epsilon}^{2}$, respectively.

Remark. $S_{\epsilon}^{2}$ is the sphere of radius $1 / \sqrt{\epsilon}$. Since any geodesic triangle $T^{*} \subset S_{\epsilon}^{2}$, must lie in some hemisphere, we see that the perimeter of $T^{*}$ can be no larger than $2 \pi / \sqrt{\epsilon}$ (the circumference of the equator). So, when $\epsilon>0$, the $C A T(\epsilon)$-inequality only makes sense for triangles of perimeter $\leq 2 \pi / \sqrt{\epsilon}$.

Now let $(X, d)$ be a metric space. A path $\alpha:[a, b] \rightarrow X$ is a geodesic if it is an isometric embedding, i.e., if $d(\alpha(t), \alpha(s))=|t-s|$ for all $s, t$ in $[a, b]$.

Definition 1.2. A metric space $X$ is a geodesic space (or a "length space") if an two points can be connected by a geodesic segment.

We shall also assume that $X$ is complete and locally compact. (The hypothesis of local compactness could be removed, cf., [BH].)

The notion of a geodesic triangle clearly makes sense in a geodesic space as does the $C A T(\epsilon)$-inequality.

Definition 1.3. A geodesic space $X$ is $C A T(\epsilon)$ if the $C A T(\epsilon)$-inequality holds for all geodesic triangles $T$ of perimeter $\leq 2 \pi / \sqrt{\epsilon}$ and for all choices of vertex $x$ and point $y$ on the opposite edge. (The condition that the perimeter be $\leq 2 \pi / \sqrt{\epsilon}$ is interpreted to be vacuous if $\epsilon \leq 0$.) $X$ has curvature $\leq \epsilon$, written $K(X) \leq \epsilon$, if it satisfies $C A T(\epsilon)$ locally.

Remarks. (i) If $\epsilon^{\prime}<\epsilon$, then $C A T\left(\epsilon^{\prime}\right)$ implies $C A T(\epsilon)$ and $K(X) \leq \epsilon^{\prime}$ implies $K(X) \leq \epsilon$

(ii) There is a completely analogous definition of curvature bounded from below: one simply reverses the $C A T(\epsilon)$-inequality. (See $[\mathrm{ABN}]$.)

Some consequences of $C A T(\epsilon)$.

(i) There are no digons in $X$ of perimeter $<2 \pi / \sqrt{\epsilon}$. (A digon is a configuration consisting of two distinct geodesic segments between points $x$ and $y$.) The reason 
a triangle for which the $C A T(\epsilon)$-inequality clearly fails. As special cases of this principle, we have the following.

a) If $X$ is $C A T(1)$, then a geodesic between two points of distance $<\pi$ is unique.

b) If $X$ is $C A T(1)$, then there is no closed geodesic of length $<2 \pi$. (A closed geodesic is an isometric embedding of a circle.)

c) If $X$ is $C A T(0)$, then any two points are connected by a unique geodesic.

(ii) If $X$ is $C A T(0)$, then the distance function $d: X \times X \rightarrow[0, \infty)$ is convex. (In general, a function $\varphi: Y \rightarrow \mathbb{R}$ on a geodesic space $Y$ is convex, if given any geodesic path $\alpha:[a, b] \rightarrow Y$ the function $\varphi \circ \alpha:[a, b] \rightarrow \mathbb{R}$ is a convex function. In particular, $X \times X$, with the product metric, is a geodesic space and the statement that $d: X \times X \rightarrow[0, \infty)$ is convex means that given geodesic paths $\alpha:[a, b] \rightarrow X$ and $\beta:[c, d] \rightarrow X$ the function $(s, t) \rightarrow d(\alpha(s), \beta(t))$ is a convex function on $[a, b] \times[c, d]$.

There is the following generalization of the Cartan-Hadamard Theorem for nonpositively curved manifolds.

Proposition 1.4. If $X$ is a geodesic space with convex distance function (e.g., if $X$ is $C A T(0)$ ), then $X$ is contractible.

Proof. The convexity of the distance function implies that $X$ has no digons. Hence, any two points of $X$ are connected by a unique geodesic. Choose a base point $x_{0}$ and define the contraction $H: X \times I \rightarrow X$ by contracting along the geodesic to $x_{0}$. The proof that $H$ is continuous follows easily from the convexity of $d$.

Remark. Suppose $K(X) \leq \epsilon$. Then since $C A T(\epsilon)$ holds locally, $X$ is locally convex (i.e., in any sufficiently small open set, any two points are connected by a unique geodesic). Therefore, $X$ is locally contractible. In particular, any such $X$ has a universal cover.

Theorem 1.5. Let $\epsilon \leq 0$. If $X$ is a geodesic space with $K(X) \leq \epsilon$, then its universal cover $\tilde{X}$ is $C A T(\epsilon)$. (In particular, $\tilde{X}$ is contractible.)

This theorem is stated by Gromov in [G, p. 119] and proved in W. Ballman's article in [GH, p. 193]. (Quite possibly, it was known to Aleksandrov.)

Remark. Theorem 1.5 is not true for $\epsilon>0$. There is an analogous result for $\epsilon>0$ : the hypothesis of simple connectivity is unimportant, but one needs to rule out the possibility of closed geodesics of length $<2 \pi / \sqrt{\epsilon}$. A version of this is stated as Lemma 3.1, below.

Corollary 1.6. If $K(X) \leq 0$, then $X$ is a $K(\pi, 1)$-space (i.e., $X$ is aspherical).

\section{Piecewise constant curvature polyhedra.}

Let $M_{\epsilon}^{n}$ stand for $S_{\epsilon}^{n}, \mathbb{E}^{n}$ or $\mathbb{H}_{\epsilon}^{n}$ as $\epsilon$ is greater than, equal to, or less than 0 , respectively. A "half-space" in $S_{\epsilon}^{n}$ is a hemisphere; a "half-space" in $\mathbb{E}^{n}$ or $\mathbb{H}_{\epsilon}^{n}$ has its usual meaning.

Definition 2.1. A (convex) cell in $M_{\epsilon}^{n}$ is a compact intersection of a finite number of half-spaces. (When $\epsilon>0$, one can also require that the cell does not contain a pair of antipodal points.) 
Definition 2.2. An $M_{\epsilon}$ cell complex $X$ is a cell complex formed by gluing together cells in $M_{\epsilon}^{n}$ via isometries of their faces. ( $\epsilon$ is fixed, $n$ can vary.) If $\epsilon=0, X$ is called piecewise Euclidean (abbreviated $P E$ ). If $\epsilon=1, X$ is piecewise spherical (abbreviated $P S$ ). If $\epsilon=-1, X$ is piecewise hyperbolic (abbreviated $P H$ ).

Example 2.3. The surface of a cube is a $P E$ complex.

If $X$ is an $M_{\epsilon}$ cell complex, then we can measure the length $\ell$ of a path in $X$ : the length of the portion of the path within a given cell is defined using arc length in $M_{\epsilon}^{n}$. The intrinsic metric $\ell$ on $X$ is defined as follows:

$$
d(x, y)=\inf \{\ell(\alpha) \mid \alpha \text { is a path from } x \text { to } y\}
$$

(If $X$ is not path connected, the $d$ may take $\infty$ as a value.)

Does the intrinsic metric give $X$ the structure of a geodesic space? The issue is whether the infimum occurring in the definition of $d$ can actually be realized by a minimal path. If $X$ is locally finite and if there is a $\delta>0$ so that all closed $\delta$-balls in $X$ are compact (e.g., if $X$ is a finite complex), then the Arzela-Ascoli Theorem implies that $X$ is a complete geodesic space.

Links. Suppose that $\sigma$ is an $n$-cell in $M_{\epsilon}^{n}$ and that $v$ is a vertex of $\sigma$. The Riemannian metric on $M_{\epsilon}^{n}$ gives an inner product on its tangent space $T_{v}\left(M_{\epsilon}^{n}\right)$ at $v$. The set of inward pointing directions at $v$ is subset of the unit sphere in $T_{v}\left(M_{\epsilon}^{n}\right)$. In fact, this subset is a spherical cell, which we denote by $L k(v, \sigma)$. We think of it as a cell in $S^{n-1}$, well-defined up to isometry.

Figure 2

If $X$ is an $M_{\epsilon}$-complex and $v$ is a vertex of $X$, then the link of $v$ in $X$ is defined by

$$
L k(v, X)=\bigcup_{v \in \sigma} L k(v, \sigma)
$$

This is a $P S$ cell complex. Thus, the link of a vertex in any $M_{\epsilon}$ cell complex has a natural piecewise spherical structure.

Example 2.4. As in 2.3, let $X$ be the surface of a cube and $v$, a vertex. Then the link of $v$ in each square is a circular arc of length $\pi / 2$; hence, the link of $v$ in $X$ is a circle of length $3 \pi / 2$.

In [G, p. 120] Gromov gave the following "infinitesimal" condition for deciding if a piecewise constant curvature polyhedron has curvature bounded from above.

Theorem 2.5. (Aleksandrov, Gromov, Ballman) Suppose $X$ is an $M_{\epsilon}$-cell complex. Then $K(X) \leq \epsilon$ if and only if for each vertex $v, L k(v, X)$ is $C A T(1)$.

A proof of this can be found in Ballman's article in [GH; p. 197]. The result must have also been known to Aleksandrov's school, since they knew that an " $M_{\epsilon}$ 
Example 2.6. A $P S$ structure on a circle $S$ is $C A T(1)$ if and only if $\ell(S) \geq 2 \pi$. Therefore, a $P E$ structure on a surface has $K \leq 0$ if and only if at each vertex the sum of the angles is $\geq 2 \pi$. For example, the surface of a cube does not have nonpositive curvature.

\section{$\S 3$. The $C A T(1)$ condition for links.}

In order to use Theorem 2.5 we need to be able to decide when the link of a vertex is $C A T(1)$. So, suppose $L$ is some $P S$ cell complex. We need to be able to answer the following.

Question. How do you tell if $L$ is $C A T(1)$ ?

The following lemma gives an inductive procedure for studying this question.

Lemma 3.1. A PS complex $L$ is $C A T(1)$ if and only if

(i) $K(L) \leq 1$, and

(ii) every closed geodesic in $L$ has length $\geq 2 \pi$.

By Theorem 2.5, condition (i) can be checked by looking at links of vertices in $L$. Thus, (ii) is the crucial condition.

We next would like to explain several situations in which we have a satisfactory answer to our question. These will be grouped under the following headings:

a) Gromov's Lemma

b) Moussong's Lemma

c) Orthogonal joins

d) Spherical buildings

e) Polar duals of hyperbolic cells

f) Branched covers of round spheres

In these notes we will mostly be concerned with the first two headings, (and we will confine ourselves to a few brief comments about the other four).

a) Gromov's Lemma. Let $\square^{n}$ denote a regular $n$-cube in $\mathbb{E}^{n}$ and let $v$ be a vertex of $\square^{n}$. Then $L k\left(v, \square^{n}\right)$ is the regular spherical $(n-1)$-simplex $\Delta^{n-1}$ spanned by the standard basis $e_{1}, \cdots, e_{n}$ of $\mathbb{R}^{n}$. (So, $\Delta^{n-1}$ is the intersection of $S^{n-1}$ with the positive "quadrant" $[0, \infty)^{n}$ in $\left.\mathbb{R}^{n}\right)$.

A spherical $(n-1)$-simplex isometric to $\Delta^{n-1}$ will be called an all right simplex.

An all right simplex is characterized by the fact that all its edge lengths are $\pi / 2$. Alternatively, it can be characterized by the fact that all its dihedral angles are $\pi / 2$.

Definition 3.2. A $P S$ simplicial cell complex is all right if each of its simplices is all right.

Example 3.3. If $X$ is a $P E$ cubical complex, then each of its links is an all right simplicial cell complex.

Definition 3.4. A simplicial complex $K$ is a flag complex if any finite set of vertices, which are pairwise joined by edges, span a simplex in $K$.

Combinatorialists use "clique complex" instead of "flag complex". Alternative terminology, which has been used elsewhere, is that $K$ is "determined by its 1skeleton", or $K$ has "no empty simplices", or $K$ satisfies the "no $\Delta$-condition". 
Remark 3.5. Let $V$ be a set with a symmetric and reflexive relation (an "incidence relation"). Let $K$ be the abstract simplicial complex whose simplices are the finite subsets of $V$ which are pairwise related. Then $K$ is a flag complex. Conversely, given a flag complex $K$, one defines a relation on its vertex set $V$ by saying that two vertices are related if they are joined by an edge. This relation gives back $K$ as its associated complex.

Example 3.6. Let $\mathcal{P}$ be a poset. Then if we make the order relation symmetric and take the associated simplicial complex, we get a flag complex. Its poset of simplices is denoted by $\mathcal{P}^{\prime}$, and called the derived complex of $\mathcal{P}$. The elements of $\mathcal{P}^{\prime}$ are finite chains $\left(v_{0}<\cdots<v_{k}\right)$ in $\mathcal{P}$.

Example 3.7. If $\mathcal{P}$ is the poset of cells in a cell complex, then $\mathcal{P}^{\prime}$ can be identified with the poset of simplices in its barycentric subdivision. Thus, the barycentric subdivision of any cell complex is a flag complex.

Example 3.8. If $K$ is the boundary of an $m$-gon (i.e., $K$ is a circle with $m$ edges) then $K$ is a flag complex if and only if $m>3$.

Lemma 3.9. (Gromov's Lemma) Let $L$ be an all right, $P S$ simplicial complex. Then $L$ is $C A T(1)$ if and only if it is a flag complex.

Corollary 3.10. (Berestovskii [Ber]) Any polyhedron has a PS structure which is $C A T(1)$.

Proof. Let $L$ be a cell complex. By taking the barycentric subdivision we may assume that $L$ is a flag complex. Then give $L$ a piecewise spherical structure by declaring each simplex to be all right.

Corollary 3.11. Let $X$ be a PE cubical complex. Then $K(X) \leq 0$ if and only if the link of each vertex is a flag complex.

Application 3.12. (Hyperbolization). In [G] Gromov described several functorial procedures for converting a cell complex $J$ (usually a simplicial or cubical complex) into a $P E$ cubical complex $\mathcal{H}(J)$ with nonpositive curvature. (See also [DJ] and [CD2].) $\mathcal{H}(J)$ is called a "hyperbolization" of $J$. Since $\mathcal{H}(J)$ is aspherical it cannot, in general, be homeomorphic to $J$. However, there is a natural surjection $\mathcal{H}(J) \rightarrow J$ Also, $\mathcal{H}(J)$ should have the same local structure as $J$ in the following sense: the link of each "hyperbolized cell" is $P L$ homeomorphic to the link of the corresponding cell in $J$. Usually, the new link will be the barycentric subdivision of the old one (or else the suspension of a barycentric subdivision of an old link). Thus, the new links will be flag complexes and Gromov's Lemma can be used to prove that $\mathcal{H}(J)$ is nonpositively curved. (A different argument is given in [DJ]] and [G].)

The proof of Gromov's Lemma is based on the following.

Sublemma 3.13. ([G, p.122]). Let $v$ be a vertex in an all right, $P S$ simplicial complex and let $B$ be the closed ball of radius $\pi / 2$ about $v$ (i.e., $B$ is the closed star of $v$ ). Let $x, y$ be points in $\partial B$ (the sphere of radius $\pi / 2$ about $v$ ) and let $\gamma$ be a geodesic segment from $x$ to $y$ such that $\gamma$ intersects the interior of $B$. Then 
Figure 3

Proof. Let $\Delta$ be an all right simplex in $B$ with one vertex at $v$ and suppose that $\gamma$ intersects the interior of $\Delta$. Consider the union of all geodesic segment which start at $v$, pass through a point in $\gamma \cap \Delta$ and end on the face of $\Delta$ opposite to $v$. It is an isoceles spherical 2-simplex with two edges of length $\pi / 2$. (Think of a spherical 2 -simplex with one vertex at the north pole and the other two on the equator.) Let $\Omega$ be the union of all these 2 -simplices. Then $\Omega$ can be "developed" onto the northern hemisphere of $S^{2}$. If $\ell(\gamma)<\pi$, then $\Omega$ is isometric to a region of $S^{2}$ so that $v$ maps to the north pole and $x$ and $y$ to points on the equator. But if two points on the equator of $S^{2}$ are of distance $<\pi$, then the geodesic between them is a segment of the equator. This contradicts the hypothesis that the image of $\gamma$ intersects the open northern hemisphere.

Proof of Gromov's Lemma. Let $L$ be an all right, $P S$ simplicial cell complex. First suppose that $L$ is not a flag complex. Then either $L$ or the link of some simplex of $L$ contains an "empty" triangle. Such a triangle is a closed geodesic of length $3 \pi / 2$ (which is $<2 \pi$ ). Hence, $L$ is not $C A T(1)$.

Conversely, suppose that $L$ is a flag complex. Then the link of each vertex is also a flag complex and by induction on dimension we may assume that $K(L) \leq 1$. Hence, it suffices to show every closed geodesic in $L$ has length $\geq 2 \pi$. Suppose, to the contrary, that $\alpha$ is a closed geodesic with $\ell(\alpha)<2 \pi$. Let $L^{\prime}$ be the full subcomplex of $L$ spanned by the set of vertices $v$ such that $\alpha \cap \operatorname{Star}(v) \neq \emptyset$. (Here $\operatorname{Star}(v)$ denotes the open star of $v$.) By Sublemma 3.13, $\alpha$ cannot intersect two disjoint open stars. Hence any two vertices of $L^{\prime}$ must be connected by an edge. Since $L$ is a flag complex, this implies $L^{\prime}$ is an all right simplex. But this is impossible since a simplex contains no closed geodesic.

\section{b) Moussong's Lemma.}

Definition 3.14. A spherical simplex has size $\geq \pi / 2$ if all of its edge lengths are $\geq \pi / 2$.

Definition 3.15. Let $L$ be a $P S$ simplicial complex with simplices of size $\geq \pi / 2$. $L$ is a metric flag complex if given a set of vertices $\left\{v_{0}, \cdots, v_{k}\right\}$, which are pairwise joined by edges, such that there exists a spherical $k$-simplex with these edge lengths, then $\left\{v_{0}, \cdots, v_{k}\right\}$ spans a $k$-simplex in $L$.

Lemma 3.16. (Moussong's Lemma) Let L be a PS simplicial complex with simplices of size $\geq \pi / 2$. Then $L$ is $C A T(1)$ if and only if it is a metric flag complex.

This generalization of Gromov's Lemma is the main technical result in the Ph.D. 
Gromov's Lemma and we will not try to explain it here. We will use it in the next chapter to show that a certain $P E$ complex associated to any Coxeter group is $C A T(0)$.

Definition 3.17. A cell is simple if the link of each vertex is a simplex.

The edge lengths of such a simplex are interior angles in the 2-dimensional faces. Thus, such a simplex has size $\geq \pi / 2$ if all such angles in the 2 -cells are $\geq \pi / 2$.

Corollary 3.18. Let $X$ be a PE complex with simple cells and with 2-cells having nonacute angles. Then $K(X) \leq 0$ if and only if the link of each vertex is a metric flag complex.

c) Orthogonal joins. Suppose that $\sigma_{1} \subset S^{k_{1}}$ and $\sigma_{2} \subset S^{k_{2}}$ are spherical cells. Regard $S^{k_{1}}$ and $S^{k_{2}}$ as a pair of orthogonal great subspheres in $S^{k_{1}+k_{2}+1} \subset \mathbb{R}^{k_{1}+1} \times$ $\mathbb{R}^{k_{2}+1}$. Then the orthogonal join $\sigma_{1} * \sigma_{2}$ of $\sigma_{1}$ and $\sigma_{2}$ is the union of all geodesic segments from $\sigma_{1}$ to $\sigma_{2}$ in $S^{k_{1}+k_{2}+1}$. It is naturally a spherical cell of dimension equal to $\operatorname{dim} \sigma_{1}+\operatorname{dim} \sigma_{2}+1$. If $L_{1}$ and $L_{2}$ are $P S$ cell complexes, then their orthogonal join $L_{1} * L_{2}$ is defined to be the union of all cells $\sigma_{1} * \sigma_{2}$ where $\sigma_{1}$ is a cell in $L_{1}$ and $\sigma_{2}$ is a cell in $L_{2}$. It is naturally a $P S$ cell complex, homeomorphic to the usual topological join of the underlying polyhedra.

The following result is proved in the Appendix of [CD1].

Proposition 3.19. If $L_{1}$ and $L_{2}$ are $C A T(1), P S$ cell complexes, then $L_{1} * L_{2}$ is $C A T(1)$.

Remark 3.20. For example, taking $L_{2}$ to be a point we see if $L_{1}$ is $C A T(1)$, then so is the "spherical cone" on $L_{1}$. Similarly, taking $L_{2}=S^{0}$, we see that the "spherical suspension" of $L_{1}$ is $C A T(1)$.

d) Spherical buildings. Tits has defined a certain remarkable class of simplicial complexes called "buildings", e.g., see [Br2] and [R]. Associated to a building $B$ there is a Coxeter group $W$. (This will be defined in Chapter II.) The building $B$ can be written as a union of apartments $A_{\alpha}$,

$$
B=\bigcup A_{\alpha}
$$

where each $A_{\alpha}$ is isomorphic to the Coxeter complex for $W$. If $W$ is a finite group, then this Coxeter complex can naturally be thought of as a triangulation of $S^{n}$, the round $n$-sphere, for some $n$. The building is called spherical if its associated Coxeter group is finite (so that each apartment is a round sphere). Thus, a spherical building has a natural structure of a $P S$ simplicial complex.

The axioms for buildings imply that any two points in $B$ lie in a common apartment. Furthermore, (at least when $B$ is spherical) the geodesic between them also lies in this apartment. From this we can immediately deduce the following. (See also [D4].)

Theorem 3.21. Any spherical building is $C A T(1)$.

Example 3.22. A generalized $m$-gon is a connected, bipartite graph of diameter $m$ and girth $2 m$. (A graph is bipartite if its vertices can be partitioned into two sets so that no two vertices in different sets span an edge. The diameter of a graph 
a circuit.) A 1-dimensional spherical building is the same thing as a generalized $m$-gon $(m \neq \infty)$. The piecewise spherical structure is defined by declaring each edge to have length $\pi / m$.

e) Polar duals of hyperbolic cells. Suppose that $C^{n}$ is a convex $n$-cell in hyperbolic $n$-space $\mathbb{H}^{n}$. Let $F$ be a face of codimension $k$ in $C^{n}, k \geq 1$. Choose a point $x$ in the relative interior of $F$ and consider the unit sphere $S^{n-1}$ in the tangent space $T_{x} \mathbb{H}^{n}$. The set of outward-pointing unit normals to the codimension one faces which contain $F$ span a spherical $(k-1)$-cell in $S^{n-1}$ which we denote by $\sigma_{F}$. (Roughly, $\sigma_{F}$ is the set of all outward pointing unit normals at $F$.) The polar dual of $C^{n}$ is defined as

$$
P\left(C^{n}\right)=\bigcup_{F} \sigma_{F}
$$

It is a $P S$ cell complex, which, it is not difficult to see, is homeomorphic to $S^{n-1}$. For further details, see [CD4].

Remark 3.23. (i) The same construction can be carried out in $\mathbb{E}^{n}$ or $S^{n}$. For a cell in $\mathbb{E}^{n}$ its polar dual is a $P S$ cell complex which is isometric to the round $(n-1)$ sphere. For a cell $C^{n}$ in $S^{n}$, its polar dual is just the boundary of the dual cell $C^{*}$, where $C^{*}=\left\{x \in S^{n} \mid d\left(x, C^{n}\right) \geq \pi / 2\right\}$. In all three cases, the cell structure on $P\left(C^{n}\right)$ is combinatorially equivalent to the boundary to the dual polytope to $C^{n}$.

(ii) If we use the quadratic form model for $\mathbb{H}^{n}$, and $C^{n} \subset \mathbb{H}^{n}$, then $P\left(C^{n}\right)$ is naturally a subset of the unit pseudosphere, $S_{1}^{n}=\left\{x \in \mathbb{R}^{n+1} \mid\langle x, x\rangle=1\right\}$, where $\langle x, x\rangle=-\left(x_{1}\right)^{2}+\left(x_{2}\right)^{2}+\cdots+\left(x_{n+1}\right)^{2}$.

Theorem 3.24. Suppose $C^{n}$ is a convex cell in $\mathbb{H}^{n}$. Then $P(C)$ is $C A T(1)$.

When $n=2, P\left(C^{2}\right)$ is a circle, the length of which is the sum of the exterior angles of $C^{2}$. By the Gauss-Bonnet Theorem, this sum is $2 \pi+\operatorname{Area}\left(C^{2}\right)$. This completes the proof for $n=2$. When $n=3$, the theorem is due to Rivin and Hodgson $[\mathrm{RH}]$. For $n>3$, it appears in [CD4].

Further Remarks 3.25. (i) A stronger result is actually true. The length of any closed geodesic in $P\left(C^{n}\right)$ is strictly greater than $2 \pi$. (As we saw, for $n=2$, this follows from the Gauss-Bonnet Theorem.) Furthermore, the same is true for the link of every cell in $P\left(C^{n}\right)$ (since such a link is, in fact, the polar dual of some face of $C^{n}$.) Sometimes I have defined a $P S$ cell complex to be "large" if it is $C A T(1)$. Perhaps $P S$ complexes satisfying the above stronger condition should be "extra large".

(ii) The definition of polar dual makes sense for any intersection of half-spaces in $\mathbb{H}^{n}$ (compact or not) and it is proved in [CD4] that these polar duals are also $C A T(1)$.

(iii) The main argument of $[\mathrm{RH}]$ is in the converse direction. They show that any $P S$ structure on $S^{2}$ which is extra large arises as the polar dual of a 3 -cell in $\mathbb{H}^{3}$ (unique up to isometry). An analogous result, relating metrics with $K \geq 1$ on $S^{2}$ to convex surfaces in $\mathbb{E}^{3}$ had been proved much earlier by Aleksandrov.

(iv) My main interest in Theorem 3.24 is that it provides a method for constructing a large number of examples of $C A T(1), P S$ structures on $S^{n-1}$, which are not covered by Moussong's Lemma. Moreover, if we deform a convex cell in $\mathbb{H}^{n}$ we 
(v) A nice application of Theorem 3.24 is given in $[\mathrm{CDM}]$. Identify $\mathbb{H}^{n}$ with the sheet of the hyperboloid in $\mathbb{R}^{n+1}$ defined by $\langle x, x\rangle=-1$ and $x_{n+1}>0$. Let $V$ be any discrete subset of $\mathbb{H}^{n}$ such that each Dirichlet domain for $V$ is bounded. Take the convex hull of $V$ in $\mathbb{R}^{n+1}$ and let $B(V)$ denote its boundary. It is not hard to see that the restriction of the bilinear form to the tangent space of any face in $B(V)$ is positive definite and hence, that $B(V)$ has a natural $P E$ structure. Moreover, for any vertex $v$ in $V$, the link of $v$ in $B(V)$ can be identified with the polar dual of the Dirichlet cell centered at $v$. So, by Theorem 3.24, $B(V)$ is CAT (0). As a corollary we have that any complete hyperbolic manifold has a nonpositively curved, $P E$ structure.

\section{f) Branched covers of round spheres.}

Suppose that $M^{n}$ is a smooth Riemannian manifold and that $p: \tilde{M}^{n} \rightarrow M^{n}$ is a branched covering by some other manifold $\tilde{M}^{n}$. The metric on $M^{n}$ induces a (non-Riemannian) metric on $\tilde{M}^{n}$.

Question. If $M^{n}$ has sectional curvature $\leq \epsilon$, then when is $K\left(\tilde{M}^{n}\right) \leq \epsilon$ ?

We further suppose that the branching is locally modeled on $\mathbb{R}^{n} \rightarrow \mathbb{R}^{n} / G$ where $G$ is some finite linear group. (Since $M^{n}$ is a manifold we must therefore have that $\mathbb{R}^{n} / G$ is homeomorphic to $\mathbb{R}^{n}$.)

The following two conditions are easily seen to be necessary for $K(\tilde{M}) \leq \epsilon$ :

(i) $K(M) \leq \epsilon$

(ii) locally, the closure of each stratum of the branched set is a convex subset of $M$.

Let $x \in M$ be a branch point and let $S_{x}$ be the unit sphere in $T_{x} M$. There is an induced finite sheeted branched cover $\tilde{S}_{x} \rightarrow S_{x}$. Since the branched set in $S_{x}$ must satisfy (ii), it follows that the metric on $\tilde{S}_{x}$ (induced from the round metric on $S_{x}$ ) is piecewise spherical. We think to $\tilde{S}_{x}$ as the "link" at a point $\tilde{x} \in p^{-1}(x)$. It turns out ([CD1, Theorem 5.3]) that together with (i) and (ii) the following condition is necessary and sufficient for $K \tilde{M})$ to be $\leq \epsilon$ :

(iii) $\tilde{S}_{x}$ is $C A T(1)$, for all branch points $x$.

Therefore, the answer to our question is closely tied to the question of when the branched cover of a round sphere is $C A T(1)$. A detailed study of this question is made in [CD1].

For example, suppose that $G$ is a finite, noncyclic subgroup of $S O(3)$ (so that $G$ is either dihedral or the group of orientation-preserving symmetries of a regular solid). Then $S^{2} / G$ is homeomorphic to $S^{2}$ and $S^{2} \rightarrow S^{2} / G$ has three branch points. Choose three points $x_{1}, x_{2}$ and $x_{3}$ in the round 2-sphere $S^{2}$ and assign $x_{i}$ a branching order of $m_{i}$, where $\Sigma\left(1 / m_{i}\right)>1$. Let $\tilde{S}^{2} \rightarrow S^{2}$ be the corresponding $|G|$-fold branched cover. In [CD1] we prove the following result.

Proposition 3.26. $\tilde{S}^{2}$ is $C A T(1)$ if and only if

(i) $x_{1}, x_{2}$, and $x_{3}$ lie on a great circle in $S^{2}$, but are not contained in any semicircle, and

(ii) $d\left(x_{i}, x_{j}\right) \geq \pi / m_{k}$, where $(i, j, k)$ is any permutation of $(1,2,3)$.

\section{Infinitesimal shadows and the ideal boundary.}

Suppose that $X$ is a piecewise constant curvature polyhedron and that 
geodesic arcs $c_{\text {out }}:[0, a) \rightarrow X$ and $c_{\text {in }}:[0, a) \rightarrow X$, defined by $c_{\text {out }}(t)=c(t)$ and $c_{\mathrm{in}}(t)=c(-t)$, and two unit tangent vectors $c_{\mathrm{out}}^{\prime}$ and $c_{\mathrm{in}}^{\prime}$ in $L k(c(0), X)$. The proof of the following lemma is left as a straightforward exercise for the reader.

Lemma 4.1. A piecewise geodesic path $c:(-a, a) \rightarrow X$ is a local geodesic at $c(0)$ (i.e., its restriction to some smaller interval about 0 is a geodesic) if and only if the distance from $c_{\mathrm{in}}^{\prime}$ to $c_{\mathrm{out}}^{\prime}$ in $\operatorname{Lk}(c(0), X)$ is $\geq \pi$.

Example 4.2. Let $S^{1}(2 \pi+\delta)$ denote a circle of circumference $2 \pi+\delta$, and let $X$ denote the Euclidean cone on $S^{1}(2 \pi+\epsilon)$. Thus, $X=\left([0, \infty) \times S^{1}(2 \pi+\delta)\right) / \sim$, where $(r, \theta) \sim\left(r^{\prime}, \theta^{\prime}\right)$ if and only if $r=r^{\prime}=0$. The metric is given by the usual formula for the metric on $\mathbb{R}^{2}$ in polar coordinates (see [BH]). Let $\theta_{1}, \theta_{2} \in S^{1}(2 \pi+\delta$ ) be two points with $d\left(\theta_{1}, \theta_{2}\right) \geq \pi$ and consider the path $c: \mathbb{R} \rightarrow X$ defined by

$$
c(t)= \begin{cases}\left(-t, \theta_{1}\right) ; & \text { for } t \leq 0 \\ \left(t, \theta_{2}\right) ; & \text { for } t \geq 0\end{cases}
$$

It follows from 4.1, that $c$ is a geodesic in $X$. Thus, if $\delta>0$, there are many possible ways to continue the geodesic ray $\left.c\right|_{(-\infty, 0]}$ to a geodesic line. These continuations are parametrized by an arc of $\theta_{2}$ 's, in fact, by the arc of radius $\frac{1}{2} \delta$ about the point of distance $\pi+\frac{1}{2} \delta$ from $\theta_{1}$.

This example illustrates a dramatic difference between singular metric spaces (e.g., piecewise constant curvature polyhedra) and Riemannian manifolds: in the singular case extensions of geodesic segments are not necessarily unique.

Suppose that $X$ is a piecewise constant curvature polyhedron, that $x \in X$ and that $v \in L k(x, X)$. The infinitesimal shadow of $x$ with respect to $v$, denoted by Shad $(x, v)$, is the subset of $L k(x, X)$ consisting of all $w \in L k(x, X)$ such that there is a goedesic $c:(-a, a) \rightarrow X$ with $c(0)=x, c_{\text {in }}^{\prime}=v$ and $c_{\text {out }}^{\prime}=w$. Thus, Shad $(x, v)$ measures the possible outgoing directions of possible extensions for a geodesic coming into $x$ from the direction $v$. In particular, $X$ has "extendible geodesics" if and only if Shad $(x, v)$ is nonempty for all choices of $x$ and $v$.

For example, if $\operatorname{Lk}(x, X)$ is isometric to the round sphere $S^{n-1}$, then it follows from Lemma 4.1 that the infinitesimal shadow of $x$ with respect to $v$ is the antipodal point $-v$.

As another example, suppose that $X$ is the cone on $S^{1}(2 \pi+\delta), \delta \geq 0$, as in Example 4.2. Then any infinitesimal shadow at the cone point is an arc of radius $\frac{1}{2} \delta$ in $S^{1}(2 \pi+\delta)$. Combining this example with Lemma 4.1, we get the following result.

Lemma 4.3. Suppose that $X$ is a piecewise constant curvature polyhdedron, that $x \in X$ and that $v \in \operatorname{Lk}(x, X)$. Then

$$
\operatorname{Shad}(x, v)=\operatorname{Lk}(x, X)-B_{\pi}(v)
$$

where $B_{\pi}(v)$ denotes the open ball in $L k(x, X)$ of radius $\pi$ about $v$.

The ideal boundary. Now suppose that $X$ is a proper CAT (0) space. ("Proper" means that closed metric balls are compact.) Then $X$ can be compactified to a space $\bar{X}$ by adding an "ideal boundary" $X(\infty)$. Here is the idea.

Fix a base point $x_{0} \in X$. We compactify $X$ by adding an endpoint $c(\infty)$ to each geodesic ray $c:[0, \infty) \rightarrow X$, with $c(0)=x_{0}$. Thus, $X(\infty)$ is the set of geodesic rays 
The topology on $\bar{X}$ can be described as follows. Let $z=c(\infty) \in X(\infty)$ and let $U$ be an open neighhborhood of $c(r)$ in $S_{x_{0}}(r)$ (the sphere of radius $r$ about $x_{0}$ ) and let $V$ be the set of all points of the form $b(t), t \in(r, \infty]$, where $b:[0, \infty) \rightarrow X$ is a geodesic ray starting at $x_{0}$ and passing through $U$. The sets $V$ form a neighborhood basis at $z$. At a point $x \in X$, a neighborhood basis consists of the open balls centered at $x$.

For $s>r$, by using geodesic contraction, one can define a natural projection

$$
p: \bar{B}_{x_{0}}(s) \rightarrow \bar{B}_{x_{0}}(r)
$$

from one closed ball to a smaller one. If $c:[0, s] \rightarrow X$ is a geodesic with $c(0)=x_{0}$, then $p$ is defined by

$$
p(c(t))= \begin{cases}c(r) ; & \text { if } t \geq r \\ c(t) ; & \text { if } t \leq r\end{cases}
$$

This gives inverse systems of maps $\bar{B}_{x_{0}}(s) \rightarrow \bar{B}_{x_{0}}(r)$ and $S_{x_{0}}(s) \rightarrow S_{x_{0}}(r)$. Clearly, $X(\infty)=\lim _{\longleftarrow} S_{x_{0}}(r)$ and if $X$ has extendible geodesics, then $\bar{X}=\lim _{\longleftarrow} \bar{B}_{x_{0}}(r)$. Moreover, the topologies on $\bar{X}$ and on $X(\infty)$ are those of the inverse limits.

Example 4.4. If $X$ is a complete CAT (0) Riemannian $n$-manifold, then a geodesic ray starting at $x_{0}$ is uniquely determined by its unit tangent vector at $x_{0}$. It follows that $\bar{X}$ is homeomorphic to the $n$-disk and $X(\infty)$ to its boundary $S^{n-1}$.

A serious problem with the above definition of the ideal boundary is that it seems to depend on the base point $x_{0}$. A definition can be given which is independent of the choice of base point. One way to do this is to define an equivalence relation, asymptoty, on the set of unbased geodesic rays. Two such rays are asymptotic if they remain a bounded distance apart. One can then prove that the natural map \{rays based at $\left.x_{0}\right\} \rightarrow$ asymptoty classes of rays $\}$ is a bijection. Thus, $X(\infty)$ is the set of asymptoty classes of rays.

A third description of $\bar{X}$ and $X(\infty)$ in terms of "horofunctions" is given in [BGS]. That these definitions coincide in the Riemannian case is proved in [BGS]. The fact that the arguments of [BGS] can be extended to the general case is explained in $[\mathrm{DJ}]$ and $[\mathrm{Dr}]$ as well as in $[\mathrm{BH}]$. 


\section{Some well-known results in geometric topology and their implications for $\operatorname{CAT}(0)$ spaces.}

A closed $n$-manifold is homology sphere if it has the same homology as does $S^{n}$. Is every homology $n$-sphere homeomorphic to $S^{n}$ ? Poincaré asked this question and came up with a counterexample for $n=3$. His new homology sphere $M^{3}$ was $S^{3} / G$ where $S^{3}$ is the group of quaternions of norm one and where $G$ is the binary icosahedral group (a subgroup of order 120). To distinguish $M^{3}$ from $S^{3}$ Poincaré discovered the concept of the fundamental group and noted that $\pi_{1}\left(M^{3}\right)=G$ while $\pi_{1}\left(S^{3}\right)=1$. Later it was shown that for each $n \geq 3$ there exist homology $n$-spheres which are not simply connected. In fact, Kervaire showed in $[\mathrm{K}]$ that for $n \geq 5$ the fundamental group could be any finitely presented group $G$ satisfying $H_{1}(G)=H_{2}(G)=0$. On the other hand, the Generalized Poincaré Conjecture asserts that any simply connected homology $n$-sphere, $n>1$, is homeomorphic to $S^{n}$. For $n \geq 5$, this was proved by Smale, in the smooth case, (and then generalized to the $P L$ case by Stallings and the topological case by Newman); for $n=4$, it was proved by Freedman in $[\mathrm{F}]$.

Suppose $C^{n}$ is a compact contractible $n$-manifold with boundary. Is $C^{n}$ homeomorphic to the $n$-disk? Poincaré duality implies that $\partial C^{n}$ is a homology $(n-1)$ sphere, but there is no reason for it to be simply connected. In fact, one can prove that any homology $(n-1)$-sphere can be realized as the boundary of a contractible $n$-manifold. (The most difficult case, $n=4$, was proved in $[\mathrm{F}]$.) Hence, it follows from the previous paragraph that, for $n \geq 4$, there are examples where $C^{n}$ is not homeomorphic to the $n$-disk. (The 3 -dimensional Poincaré Conjecture asserts that there are no such examples for $n=3$.) On the other hand, for $n \geq 5$, the Generalized Poincaré Conjecture implies that $C^{n}$ is homeomorphic to the $n$-disk if and only if $\partial C^{n}$ is simply connected.

Similarly, we can ask to what extent do open contractible $n$-manifolds differ from the interior of $D^{n}$ (i.e., from $\mathbb{R}^{n}$ ). If $C^{n}$ is a compact contractible manifold, $n>2$, and if $\partial C^{n}$ is not simply connected, then the interior of $C^{n}$ is not homeomorphic to $\mathbb{R}^{n}$. The reason is that the interior of $C^{n}$ is not simply connected at infinity. (Its "fundamental group at infinity" is isomorphic to $\pi_{1}\left(\partial C^{n}\right)$.) Thus, for $n \geq 4$ there are contractible manifolds (without boundary) which are not homeomorphic to $\mathbb{R}^{n}$. This is also true for $n=3$ : there is a famous example of J. H. C. Whitehead of a contractible 3-manifold which is not simply connected at infinity. In fact, the situation is more complicated than in the compact case: the end of an open contractible manifold need not be "tame", i.e., the contractible manifold might not be homeomorphic to the interior of any compact manifold with boundary. For example, the fundamental group at infinity need not be finitely generated. On the other hand, Stallings [Sta1] (for $n \geq 5$ ) and Freedman [F] (for $n=4$ ) showed that a contractible $n$-manifold is homeomorphic to $\mathbb{R}^{n}$ if and only if it is simply connected at infinity.

Here are a few questions which we shall be concerned with.

Question 5.1. If a contractible $n$-manifold admits a cocompact transformation group, then is it homeomorphic to $\mathbb{R}^{n}$ ? In particular, if $M^{n}$ is a closed aspherical manifold, then is its universal cover homeomorphic to $\mathbb{R}^{n}$ ?

By constructing examples from reflection groups, we shall see in $\S 11$ that the answer is "no" for each $n \geq 4$. This reflection group construction suggests the 
Question 5.2. Suppose $M^{n}, n>2$, is a closed aspherical $n$-manifold with universal cover $\widetilde{M}^{n}$. If the fundamental group at infinity of $\widetilde{M}^{n}$ is finitely generated, then is it homeomorphic to $\mathbb{R}^{n}$ ? (The fundamental group at infinity $\pi_{1}^{\infty}\left(\widetilde{M}^{n}\right)$ is the inverse limit $\lim \pi_{1}\left(\widetilde{M}^{n}-K\right)$ where $K$ ranges over the compact subsets of $\widetilde{M}^{n}$.)

Something very close to an affirmative answer to this has been proved by Wright [Wr]. He shows that the inverse system $\pi_{1}\left(\widetilde{M}^{n}-K\right)$ cannot be "pro-monomorphic".

Next we turn to some questions involving nonpositively curved spaces.

Question 5.3. Suppose $X^{n}$ is a CAT (0) manifold.

a) (Gromov) Is $X^{n}$ homeomorphic to $\mathbb{R}^{n}$ ?

b) If so, is $(\bar{X}, X(\infty))$ homeomorphic to $\left(D^{n}, S^{n-1}\right)$ ? In particular, if $X^{n}$ is simply connected at infinity, then is $X(\infty)$ homeomorphic to $S^{n-1}$ ?

c) If $X(\infty)$ is a manifold, then is it homeomorphic to $S^{n-1}$ ?

The answers to all the questions in 5.3 are "no" for $n>4$ and "yes" for $n \leq 3$. Paul Thurston [Thp] has proved that the answer to a) is also "yes" when $n=4$, provided the manifold has a least one "tame point," but without this hypothesis the answer is not known. The result for $n=3$ is essentially due to Rolfsen [Ro]. For a clear discussion of these questions in dimensions 3 and 4, the reader is referred to [Thp].

Question 5.4. If $X^{n}$ is the universal cover of a nonpositively curved, closed manifold, then are the answers to Questions 5.3 the same?

A polyhedron $N^{n}$ is a $P L n$-manifold if for each $k$-cell $\sigma$ in $N, L k\left(\sigma, N^{n}\right)$ is piecewise linearly homeomorphic to $S^{n-k-1}$ (where a sphere has a standard $P L$ structure as the boundary of a convex cell).

The next result, which is proved in [DJ], asserts that in the $P L$ context, Questions 5.3 have affirmative answers.

Theorem 5.5. (Stone [Sto] and [DJ]). Suppose $X^{n}$ is a piecewise Euclidean or piecewise hyperbolic polyhedron and that

(a) $X^{n}$ is a PL manifold (i.e., the underlying polyhedral structure on $X^{n}$ is that of a PL manifold) and

(b) $X^{n}$ is $C A T(0)$.

Then $(\bar{X}, X(\infty))$ is homeomorphic to $\left(D^{n}, S^{n-1}\right)$.

The result of $[\mathrm{Sto}]$ states that $X^{n}$ is $P L$ homeomorphic to $\mathbb{R}^{n}$.

The proof of 5.5 uses the Approximation Theorem for cell-like maps due to Siebenmann $(n \geq 5)$, Quinn $(n=4)$, Armentrout $(n=3)$, and Moore $(n=2)$. Before stating this theorem, we recall some definitions. (Our discussion is taken from $[\mathrm{E}]$.)

A compact metrizable space $C$ is cell-like if there is an embedding of $C$ into the Hilbert cube $I^{\infty}$ so that for any neighborhood $U$ of $C$ in $I^{\infty}$, the space $C$ is contractible in $U$. A cell-like subspace of a manifold is cellular if it has arbitrarily small neighborhoods homeomorphic to a cell. A compact subset of $S^{n}$ is pointlike if its complement is homeomorphic to $\mathbb{R}^{n}$. A map is cell-like if each point inverse image is cell-like.

Theorem 5.6. (The Approximation Theorem) Suppose $f: M^{n} \rightarrow N^{n}$ is a cell-like map of topological manifolds. If $n=3$, further assume that $f$ is cellular (i.e., each 
A map which can be approximated by a homeomorphism is a near homeomorphism. We shall also need the following theorem of M. Brown [Bro].

Theorem 5.7. (M. Brown) An inverse limit of near homeomorphisms is a near homeomorphism.

Sketch of proof of Theorem 5.5. The rough idea is that one shows that the hypotheses of Theorem 5.5 imply that each infinitesimal shadow is cell-like. Since these shadows are basically point inverse images of the geodesic contraction map, one can then conclude from the Approximation Theorem that each closed metric ball is homeomorphic to an $n$-disk and from Brown's Theorem that

$$
(\bar{X}, X(\infty)) \cong\left(D^{n}, S^{n-1}\right)
$$

To be more explicit, let $\left(T_{n}\right)$ denote the statement of the theorem in dimension $n$ and let $\left(L_{n}\right)$ denote the following statement:

If $M^{n}$ has a $P S$ structure such that (a) $M^{n}$ is a $P L$ manifold and (b) $M^{n}$ is CAT (1), then for each $r \in(0, \pi)$ and $v \in M^{n}$ the closed metric ball $\bar{B}_{v}(r)$ is homeomorphic to $D^{n}$ and $B_{v}(\pi)$ is homeomorphic to $\mathbb{R}^{n}$.

The inductive scheme is then $\left(L_{n-1}\right) \Rightarrow\left(L_{n}\right)$ and $\left(T_{n}\right)$. For example, to see that $\left(L_{n-1}\right) \Rightarrow\left(T_{n}\right)$, let $x \in X^{n}$ and $v \in L k(x, X)$. By hypothesis, $L k(x, X) \cong S^{n-1}$ and by $\left(L_{n-1}\right), B_{v}(\pi) \cong \mathbb{R}^{n-1}$. Hence, by Lemma 4.3 , Shad $(x, v)=L k(x, X)-B_{v}(\pi)$ is pointlike. In particular, since $\operatorname{Shad}(x, v)$ is nonempty, it follows that geodesics in $X$ are extendible and from this that

$$
(\bar{X}, X(\infty)) \cong \lim _{\longleftarrow}\left(\bar{B}_{x_{0}}(r), \partial \bar{B}_{x_{0}}(r)\right)
$$

for any base point $x_{0}$. Moreover, using the fact that infinitesimal shadows are celllike it is easy to produce a cell-like map from $\bar{B}_{x_{0}}(r)$ to the disk of radius $r$ in $\mathbb{R}^{n}$. Further details can be found in Section 3 of [DJ].

A space $N$ is a homology $n$-manifold if for each $x \in N, H_{i}(N, N-x)$ vanishes for $i \neq n$ and is infinite cyclic for $i=n$. If $N$ is a polyhedron, then it is a homolgy $n$-manifold if and only if for each $k$-cell $\sigma$ of $N, L k(\sigma, N)$ has the same homology as does $S^{n-k-1}$.

Theorem 5.8. Suppose $X^{n}$ is a piecewise Euclidean or piecewise hyperbolic polyhedron and that

(a) $X^{n}$ is a homology n-manifold, and

(b) $X^{n}$ is $C A T(0)$.

Then $X$ has extendible geodesics and its ideal boundary $X(\infty)$ is a homology $(n-1)$ manifold with the same homology as $S^{n-1}$.

The point is that the arguments in the proof of Theorem 5.5 show that under the hypotheses of Theorem 5.8, each infinitesimal shadow is acyclic.

Remark 5.9. The proofs of Theorems 5.5 and 5.8 are indicative of a (conjectural) "local-to-global" principle: the topology of the ideal boundary of a CAT (0) space should be controlled by the topology of the infinitesimal shadows (reflecting the topology of the links of points). For example in Theorem 5.5, the fact that the link 
homeomorphic to $S^{n-1}$. In Theorem 5.8, the fact that the link of each vertex is a homology manifold with the same homology as $S^{n-1}$ is reflected in the fact that $X(\infty)$ has the same properties.

Here is another result (Proposition 3d.3, p. 374 of [DJ]) which clarifies the picture.

Theorem 5.10. Suppose that $X$ is a CAT(0) polyhedron such that the link of each vertex is a PL manifold (i.e., $X$ has isolated $P L$ singularities). Then any metric sphere in $X$, which does not pass through a vertex, is homeomorphic to the connected sum of the links of the vertices which it encloses and $X(\infty)$ is the inverse limit of this sequence of increasing connected sums. In particular, if $X$ is a polyhedral homology manifold (so that the link of each vertex is a PL manifold and a homology sphere), then, generically, each metric sphere is a connected sum of $P L$ homology spheres and $X(\infty)$ is the resulting inverse limit.

If a polyhedron is a topological manifold, then, of course, it is a homology manifold. Moreover, it can happen that the link of a $k$-cell, $k>0$, in a polyhedral topological manifold can be a nonsimply connected homology sphere. This is the content of the famous Double Suspension Theorem (due to Edwards in many cases and to Cannon [Ca] in complete generality). This result states that if $N^{n}$ is a $P L$ manifold and a homology sphere, then its double suspension $S^{1} * N^{n}$ is a topological manifold (which must be homeomorphic to $S^{n+2}$ by the Generalized Poincaré Conjecture). The definitive result along these lines in the following Polyhedral Manifold Characterization Theorem of [E].

Theorem 5.11. (Edwards) A polyhedral homology manifold of dimension $\geq 5$ is a topological manifold if and only if the link of each vertex is simply connected.

Thus, in spite of Theorem 5.5, the possibility remains that the answers to Questions 5.3 are negative. As we shall see in $\S 11$, this is, in fact, the case (the answers to $5.3 \mathrm{a}$ ) and b) are negative).

\section{Euler characteristics and the Combinatorial Gauss-Bonnet Theorem.}

Hopf's Conjecture. Suppose $M^{2 n}$ is a closed Riemannian manifold, with $K(M) \leq 0$. Then $(-1)^{n} \chi\left(M^{2 n}\right) \geq 0$. (Here $\chi$ denotes the Euler characteristic.)

Remark 6.1. (i) There is an analogous version of this conjecture for nonnegative curvature: the Euler characteristic should be nonnegative.

(ii) Thurston has conjectured that Hopf's Conjecture should hold for any closed, aspherical $2 n$-manifold.

The reason for believing this is the Gauss-Bonnet Theorem (proved by Chern in dimensions $>2$ ). Recall that this is the following theorem.

\section{Gauss-Bonnet Theorem.}

$$
\chi\left(M^{2 n}\right)=\int P
$$

Here $P$ is a certain $2 n$-form called the "Pfaffian" or the "Euler form". This leads to the following.

Question 6.2. Does $K\left(M^{2 n}\right) \leq 0$ imply that $(-1)^{n} P \geq 0$ ? (In other words, is 
In dimension 2 the answer is, of course, yes, since $P$ is then just the volume form times the curvature. The answer is also yes in dimension 4. A proof is given by Chern in $[\mathrm{C}]$, where the result is attributed to Milnor.

Hopf's Conjecture holds in higher dimensions under the hypothesis that the "curvature operator" is negative semi-definite (which is stronger than assuming that the sectional curvature is nonpositive). On the other hand, in dimensions $\geq 6$, Geroch [Ge] showed in 1976 that the answer to Question 6.2 is no.

The following combinatorial version of the Gauss-Bonnet Theorem is a classical result. A proof can be found in [CMS], where one can also find a convincing argument that it is the correct analog of the smooth Gauss-Bonnet Theorem.

Theorem 6.3. Suppose $X$ is a finite, PE cell complex. Then

$$
\chi(X)=\sum_{v} P(L k(v, X))
$$

Here $P$ is a certain function which assigns a real number to any finite, $P S$ cell complex. We define it below.

Let $\sigma \subset S^{k}$ be a spherical $k$-cell. Its dual cell $\sigma^{*}$ is defined by $\sigma^{*}=\{x \in$ $\left.S^{k} \mid d(x, \sigma) \geq \pi / 2\right\}$. Let $a^{*}(\sigma)$ be the volume of $\sigma^{*}$ normalized so that volume of $S^{k}$ is 1 , i.e.,

$$
a^{*}(\sigma)=\frac{\operatorname{vol}\left(\sigma^{*}\right)}{\operatorname{vol}\left(S^{k}\right)}
$$

If $L$ is a finite, $P S$ cell complex then $P(L)$ is defined by

$$
P(L)=1+\sum_{\sigma}(-1)^{\operatorname{dim} \sigma+1} a^{*}(\sigma)
$$

where the summation is over all cells $\sigma$ in $L$.

Example 6.4. . Suppose that $\sigma$ is an all right $k$-simplex. Then $\sigma^{*}$ is also an all right $k$-simplex. Since $S^{k}$ is tessellated by $2^{k+1}$ copies of $\sigma^{*}$ we see that $a^{*}(\sigma)=$ $\left(\frac{1}{2}\right)^{k+1}$. Now let $L$ be an all right $P S$ simplicial complex and $f_{i}$ the number of $i$-simplices in $L$. Then

$$
\begin{aligned}
P(L) & =1+\sum_{\sigma}\left(-\frac{1}{2}\right)^{\operatorname{dim} \sigma+1} \\
& =1+\sum_{i}\left(-\frac{1}{2}\right)^{i+1} f_{i} .
\end{aligned}
$$

The following conjecture asserts that the answer to the combinatorial version of Question 6.2 should always be yes.

Conjecture 6.5. Suppose that $L^{2 n-1}$ is a PS cell complex homeomorphic to $S^{2 n-1}$. If $L^{2 n-1}$ is $C A T(1)$, then $(-1)^{n} P\left(L^{2 n-1}\right) \geq 0$.

Thus, this conjecture implies Hopf's Conjecture for PE manifolds.

If $L$ is a flag complex, then, by Gromov's Lemma and Example 6.4, we have the 
Conjecture 6.6. Suppose that $L^{2 n-1}$ is a flag complex which triangulates $S^{2 n-1}$. Then

$$
(-1)^{n}\left(1+\sum\left(-\frac{1}{2}\right)^{i+1} f_{i}\right) \geq 0
$$

So, this conjecture implies Hopf's Conjecture for $P E$ cubical complexes which are closed manifolds.

Remark 6.7. Conjecture 6.6 is analogous to the Lower Bound Theorem in the combinatorics (a result concerning inequalities among the $f_{i}$ for simplicial polytopes). For example, the Lower Bound Theorem of [W] asserts that for any simplicial complex $L$ which triangulates $S^{3}$, we have $f_{1} \geq 4 f_{0}-10$. Conjecture 6.6 asserts that, if, in addition, $L$ is a flag complex, then $f_{1} \geq 5 f_{0}-16$. Some evidence for these conjectures is provided by the following two propositions. The first result follows from recent work of R. Stanley [St] as was observed by Eric Babson.

Proposition 6.8. Suppose that $L^{2 n-1}$ is the barycentric subdivision of the boundary complex of a convex $2 n$-cell (so that $L$ is a flag complex). Then Conjecture 6.6 holds for $L$.

Proposition 6.9. Suppose that $L^{2 n-1}$ is the polar dual of a convex cell $C^{2 n}$ in $\mathbb{H}^{n}$. Then Conjecture 6.5 holds for $L$.

This proposition follows from a formula of Hopf (predating the general GaussBonnet Theorem) which asserts the $(-1)^{n} P\left(L^{2 n-1}\right)$ is one-half the hyperbolic volume of $C^{2 n}$ (suitably normalized).

Further details about these conjectures and further evidence for them can be found in [CD3].

Remark 6.10. A natural reaction to Conjecture 6.5 is that it might contradict Geroch's result. One could try to obtain such a contradiction as follows. Take a smooth Riemannian manifold $M^{2 n}$ whose curvature operator at a point $x$ is as in Geroch's result. Then try to approximate $M^{2 n}$ near $x$ by a $P E$ cell complex with nonpositive curvature. By the main result of [CMS] the numbers $P(L)$ for $L$ a link in the complex, should approximate the Pfaffian at $x$ and hence, have the wrong sign. However, it is not clear that such an approximation exists. Thus, we are led to ask the following.

Question 6.11. Suppose $M$ is a Riemannian manifold with $K(M) \leq 0$ (we could even assume the inequality is strict). Is $M$ homeomorphic to a $P E$ cell complex $X$ with $K(X) \leq 0$ ?

As we explained in Remark $3.25(\mathrm{v})$, the answer is yes in the constant curvature case. For our conjectures to be correct, the answer, in general, should be no. 


\section{Coxeter Groups.}

Coxeter groups and Coxeter systems are defined in $\S 7$. Associated to a Coxeter system there is a simplicial complex called its "nerve". The basic result of $\S 7$ is Lemma 7.6, which asserts that any finite polyhedron can occur as the nerve of some Coxeter system. Eventually, this will be used to show that Coxeter groups provide a rich and flexible source of examples.

In $\S 8$ and $\S 9$ we discuss a beautiful, PE cell complex $\Sigma$ which is naturally associated to a Coxeter system $(W, S)$. From the results of Chapter I, we get the important result of Moussong (generalizing an earlier observation of Gromov), that $\Sigma$ is nonpositively curved and hence, contractible (since it is simply connected). The connection with reflection groups is explained in $\S 10$.

In $\S 11$ we briefly discuss some important special cases of this construction.

\section{Coxeter systems.}

Definition 7.1. Let $S$ be a finite set. A Coxeter matrix $M=\left(m_{s s^{\prime}}\right)$ is an $S \times S$ symmetric matrix with entries in $\mathbb{N} \cup\{\infty\}$ such that

$$
m_{s s^{\prime}}= \begin{cases}1 & : \text { if } s=s^{\prime} \\ \geq 2 & ; \text { if } s \neq s^{\prime}\end{cases}
$$

Definition 7.2. Given a Coxeter matrix $M=\left(m_{s s^{\prime}}\right)$, define a group $W$ by the presentation:

$$
W=\left\langle S \mid\left(s s^{\prime}\right)^{m_{s s^{\prime}}}=1, \forall\left(s, s^{\prime}\right) \in S \times S\right\rangle
$$

$W$ is called a Coxeter group.

If all the off-diagonal entries of $M$ are 2 or $\infty$, then $W$ is called right-angled.

Coxeter groups are intimately connected to the theory of reflection groups. This connection is not emphasized in this paper. For now it should suffice to mention that if a group $W$ acts properly on a connected manifold and if $W$ is generated by reflections (where a reflection is an involution whose fixed point set separates the manifold), then $W$ is a Coxeter group (cf. [D1, Theorem 4.1]).

Given $M$, it is proved in Ch. V, $\S 4.3$ of $[\mathrm{B}, \mathrm{pp} .91-92]$ that one can find a family $\left(\rho_{s}\right)_{s \in S}$ of linear reflections $\rho_{s}: \mathbb{R}^{S} \rightarrow \mathbb{R}^{S}$ so that $\rho_{s} \circ \rho_{s^{\prime}}$ has order $m_{s s^{\prime}}$ for all $\left(s, s^{\prime}\right) \in S \times S$. (Here $\mathbb{R}^{S}$ denotes the real vector space with basis $S$.) It follows that the map $s \rightarrow \rho_{s}$ extends to a representation $\rho: W \rightarrow G L\left(\mathbb{R}^{S}\right)$ called the canonical representation. The existence of this representation immediately implies the following:

a) the natural map $S \rightarrow W$ is an injection (and henceforth, we shall identify $S$ with its image in $W$ ),

b) order $(s)=2$, for all $s \in S$

c) order $\left(s s^{\prime}\right)=m_{s s^{\prime}}$, for all $\left(s, s^{\prime}\right) \in S \times S$.

The pair $(W, S)$ is called a Coxeter system.

Remark 7.3. It is proved in Ch. $\mathrm{V}, \S 4.4$ of $[\mathrm{B}, \mathrm{pp} .92-94]$ that the dual representation $\rho^{*}: W \rightarrow G L\left(\left(\mathbb{R}^{S}\right)^{*}\right)$ is faithful and has discrete image. Moreover, as explained in $\S 12, W$ acts properly on the interior of a certain convex cone in $\left(\mathbb{R}^{S}\right)^{*}$. (These results are due to Tits.) 
Lemma 7.4. ([B, p. 20]) For any $T \subset S$, the pair $\left(W_{T}, T\right)$ is a Coxeter system (i.e., its Coxeter matrix is $M \mid T$ ).

Let $(W, S)$ be a Coxeter system. We define a poset, denoted $\mathcal{S}^{f}(W, S)$ (or simply $\left.\mathcal{S}^{f}\right)$ by

$$
\mathcal{S}^{f}=\left\{T \mid T \subset S \text { and } W_{T} \text { is finite }\right\}
$$

It is partially ordered by inclusion. Consider $\mathcal{S}^{f}-\{\emptyset\}$. It is isomorphic to the poset of simplices of an abstract simplicial complex which we shall denote by $N(W, S)$ (or simply $N) . N$ is called the nerve of $(W, S)$.

In other words, the vertex set of $N$ is $S$ and a subset $T$ of $S$ spans a simplex if and only if $W_{T}$ is finite.

Example 7.5. If $W$ is finite, then $N$ is the simplex on $S$.

Which finite polyhedra occur as the nerve of some Coxeter system? The next two results show that they all do. (Compare with Corollary 3.10.)

Lemma 7.6. Let $L$ be any flag complex. Then there is a right-angled Coxeter system $(W, S)$ with $N(W, S)=L$.

Proof. Let $S$ be the vertex set of $L$ and define a Coxeter matrix $\left(m_{s s^{\prime}}\right)$ by

$$
m_{s s^{\prime}}= \begin{cases}1 & , \text { if } s=s^{\prime} \\ 2 & , \text { if }\left\{s, s^{\prime}\right\} \text { spans an edge in } L \\ \infty & , \text { otherwise }\end{cases}
$$

If $W$ is the associated right-angled Coxeter group, then $N(W, S)=L$.

In particular, since the barycentric subdivision of any (regular) cell complex is a flag complex, we have the following corollary.

Corollary 7.7. For any finite polyhedron $P$, there is a right-angled Coxeter system $(W, S)$ with $N(W, S)$ homeomorphic to $P$.

The main result of this chapter is the following theorem.

Theorem 7.8. (Gromov, Moussong) Associated to a Coxeter system $(W, S)$ there is a PE cell complex $\Sigma(W, S)(=\Sigma)$ with the following properties.

i) The poset of cells in $\Sigma$ is the poset of cosets

$$
W S^{f}=\coprod_{T \in \mathcal{S}^{f}} W / W_{T}
$$

(ii) $W$ acts by isometries on $\Sigma$ with finite stabilizers and with compact quotient.

(iii) Each cell in $\Sigma$ is simple (so that for each vertex $v, L k(v, \Sigma)$ is a simplicial cell complex). In fact, this complex is just $N(W, S)$.

(iv) $\Sigma$ is $C A T(0)$.

\section{$\S 8$. Coxeter cells.}

Throughout this section we suppose that $W$ is a finite Coxeter group. In this case, we will show that $\Sigma$ can be identified as a convex cell in $\mathbb{R}^{n}(n=\operatorname{Card}(S))$.

The canonical representation shows that $W$ can be represented as an orthogonal linear reflection group on $\mathbb{R}^{n}$. The hyperplanes of reflection divide $\mathbb{R}^{n}$ into "chambers", each of which is a simplicial cone. (See p. 85 in [B].)

Choose a point $x$ in the interior of some chamber. Define $\Sigma$ to be the convex hull of $W x$ (the orbit of $x$ ). $\Sigma$ is called a Coxeter cell of type $W$. 
Lemma 8.1. Suppose $W$ is finite.

(i) The vertex set of the Coxeter cell $\Sigma$ is $W x$.

(ii) Each face $F$ of $\Sigma$ is the convex hull of a set of vertices of the form $\left(w W_{T}\right) x$ for some $T \subset S$ and some coset $w W_{T}$ of $W_{T}$. (So, $F$ is a Coxeter cell of type $W_{T}$.)

(iii) The poset of faces of $\Sigma$ is therefore,

$$
\coprod_{T \subset S} W / W_{T}
$$

(iv) $\Sigma$ is simple cell. $L k(x, \Sigma)$ is the spherical $(n-1)$-simplex spanned by the outward pointing unit normals to the supporting hyperplanes of a chamber.

Remark 8.2. If $x$ lies in a chamber with supporting hyperplanes indexed by $S$, then the vertex set of $L k(x, \Sigma)$ is naturally identified with $S$. Moreover, the length of the edge from $s$ to $s^{\prime}$ is $\pi-\pi / m_{s s^{\prime}}$. (In other words, the corresponding angle in a 2-cell in $\Sigma$ is $\pi-\pi / m_{s s^{\prime}}$.)

Example 8.3. (i) If $W=\mathbb{Z} / 2$, then $\Sigma$ is an interval.

(ii) If $W=D_{m}$ (the dihedral group of order $2 m$ ), then $\Sigma$ is a $2 m$-gon.

(iii) If $(W, S)$ is the direct product of two Coxeter systems $\left(W_{1}, S_{1}\right)$ and $\left(W_{2}, S_{2}\right)$ (so that $W=W_{1} \times W_{2}$ and $\left.S=S_{1} \coprod S_{2}\right)$, then $\Sigma(W, S)=\Sigma\left(W_{1}, S_{1}\right) \times \Sigma\left(W_{2}, S_{2}\right)$. In particular, if $W=(\mathbb{Z} / 2)^{n}$, then $\Sigma$ is a $n$-dimensional box $(=$ the product of $n$ intervals).

(iv) If $W=S_{n}$, the symmetric group on $n$ letters, then $\Sigma$ is the $(n-1)$-cell called the permutahedron. The picture for $n=4$ is given below.

Figure 4

Remark 8.4. By choosing $x$ to be of distance 1 from each supporting hyperplane we can normalize each Coxeter cell so that every edge length is 2 .

$\S$ 9. The cell complex $\Sigma$ (in the case where $W$ is infinite).

There is an obvious way to generalize the material of the previous section to the case where $W$ is infinite. The cell complex $\Sigma$ is defined as follows. The vertex set of $\Sigma$ is $W$. Take a Coxeter cell of type $W_{T}$ for each coset $w W_{T}, T \in \mathcal{S}^{f}$. Identify the vertices of this Coxeter cell with the elements of $w W_{T}$. Identify two faces of two Coxeter cells if they have the same set of vertices. This completes the definition of $\Sigma$ as a cell complex.

If we normalize each Coxeter cell as in Remark 8.4, then the faces of the cells are identified isometrically and hence, $\Sigma$ has the structure of a $P E$ cell complex.

Remark 9.1. Let $\lambda: S \rightarrow(0, \infty)$ be a function. If, in the definition of each Coxeter 
to $s$, then the Coxeter cells again fit together to give a $P E$ structure on $\Sigma$. We have arbitrarily chosen $\lambda$ to be the constant function.

Remark 9.2. By construction, the poset

$$
W \mathcal{S}^{f}=\coprod_{T \in \mathcal{S}^{f}} W / W_{T}
$$

is the poset of cells in $\Sigma$. If $\mathcal{P}$ is any poset, then let $\mathcal{P}^{\prime}$ be its derived complex defined as in Example 3.7, (i.e., $\mathcal{P}^{\prime}$ is the poset of finite chains in $\mathcal{P}$ ). $\mathcal{P}^{\prime}$ is the poset of simplices in an abstract simplicial complex. Moreover, if $\mathcal{P}$ is the poset of cells in a cell complex, then $\mathcal{P}^{\prime}$ is the poset of simplices in its barycentric subdivision. Applying these remarks to the case at hand, we see that the barycentric subdivision $\Sigma^{\prime}$ of $\Sigma$ is just the geometric realization of $\left(W \mathcal{S}^{f}\right)^{\prime}$. Alternatively, we could have defined $\Sigma^{\prime}$ as the geometric realization of $\left(W \mathcal{S}^{f}\right)^{\prime}$ and then remarked that the poset of chains which terminate in $w W_{T}$ can naturally be identified with the set of simplices in the barycentric subdivision of a Coxeter cell of type $W_{T}$. Hence, the cellulation of $\Sigma$ by Coxeter cells could be recovered from $\Sigma^{\prime}$ by collecting together the appropriate simplices.

The link of a vertex. The group $W$ acts isometrically on $\Sigma$ and freely and transitively on its vertex set. Thus, there is an isometry of $\Sigma$ which takes any vertex onto the element $1 \in W$. What is $L k(1, \Sigma)$ (as a simplicial complex)? A cell contains the element 1 if and only if it corresponds to some identity coset $W_{T}$. Hence, the poset of simplices in $L k(1, \Sigma)$ is just $\mathcal{S}^{f}-\{\emptyset\}$, i.e.,

$$
L k(1, \Sigma)=N(W, S)
$$

What is the induced $P S$ structures on $N$ ? Two distinct vertices $s$ and $s^{\prime}$ of $N$ are connected by an edge $e_{s s^{\prime}}$ if and only if $m_{s s^{\prime}} \neq \infty$. By Remark 8.2, $\ell\left(e_{s s^{\prime}}\right)=$ $\pi-\pi / m_{s s^{\prime}}$ (where $\ell$ stands for length). Since a spherical simplex is determined by its edge lengths this determines $P S$ structure on $N$.

A proof of the following lemma can be found in [B, p.98].

Lemma 9.3. Let $T$ be a subset of $S$. Consider the $T \times T$ matrix, $\left(c_{s s^{\prime}}\right)$, where $s, s^{\prime} \in T$, and where $c_{s s^{\prime}}=\cos \left(\pi-\pi / m_{s s^{\prime}}\right)$. Then $W_{T}$ is finite if any only if $\left(c_{s s^{\prime}}\right)$ is positive definite.

Proof. Consider the canonical representation of $W_{T}$ into $G L\left(\mathbb{R}^{n}\right)$. Suppose $W_{T}$ is finite. Then we may assume that the image of this representation is contained in $O(n)$. For each $s \in T$, let $u_{s}$ be the outward-pointing unit normal to the hyperplane corresponding to $s$. Then $\left(u_{s} \cdot u_{s^{\prime}}\right)=\left(c_{s s^{\prime}}\right)$ and hence, this matrix is positive definite since $\left(u_{s}\right)_{s \in T}$ is a basis for $\mathbb{R}^{n}$. Conversely, suppose that $\left(c_{s s^{\prime}}\right)$ is positive definite. Since the canonical representation preserves the corresponding bilinear form, we get that the image of this representation is contained in $O(n)$. Since this representation is also discrete and faithful (cf. Remark 7.3), $W_{T}$ is a discrete subgroup of $O(n)$; hence, finite.

Corollary 9.4. $N(W, S)$ is a metric flag complex.

We can now prove the main result.

Proof of Theorem 7.8. We have already demonstrated the required properties of $\Sigma$ 
see that $\Sigma$ is simply connected. (One argument is to observe that the 2 -skeleton of $\Sigma$ is just the universal cover of the 2-complex associated to standard presentation of $W$.) Hence, by Theorem 1.5, it suffices to show $K(\Sigma) \leq 0$. The link of any vertex is isometric to $N$. By Corollary 9.4 and Moussong's Lemma (Lemma 3.16) $N$ is $C A T(1)$. Therefore, $K(\Sigma) \leq 0$ (by Theorem 2.5).

Remark 9.5. Theorem 7.8 was proved by Gromov [G, pp. 131-132] in the special case where $W$ is right-angled. The general case was proved in [M]. The point is that in the right-angled case $\Sigma$ is a cubical complex so we can use Gromov's Lemma (Lemma 3.9) rather than Moussong's generalization of it.

Combining Lemma 7.6 and Theorem 7.8 (in the right-angled case) we get the following.

Corollary 9.6. Given a finite flag complex $L$, there is a finite, nonpositively curved, cubical PE complex $X$ such that the link of each vertex in $X$ is isomorphic to $L$.

Proof. Let $(W, S)$ be the right-angled Coxeter system associated to $L$ and let $\Gamma$ be any torsion-free subgroup of finite index in $W$. (For example, $\Gamma$ could be the kernel of the obvious epimorphism $W \rightarrow(\mathbb{Z} / 2)^{S}$.) Set $X=\Sigma(W, S) / \Gamma$.

There is a simpler verision of the above construction, which does not directly use Coxeter groups, and which gives the following slightly more general result.

Proposition 9.7. Let $L$ be a finite simplicial complex. Then there is a finite, cubical PE complex $X$ such that the link of each vertex in $X$ is isomorphic to $L$. (Of course, by Lemma 3.9, $L$ is nonpositively curved if and only if $L$ is a flag complex.)

Proof. (Independently due to E. Babson and Bridson and Haefliger, $[\mathrm{BH}]$ ). Let $S$ be the vertex set of $L$. Consider the cube $[-1,1]^{S}$ in the Euclidean space $\mathbb{R}^{S}$ with standard basis $\left(e_{s}\right)_{s \in S}$. Let $X$ be the cubical subcomplex of $[-1,1]^{S}$ consisting of all faces parallel to a subspace of the form $\mathbb{R}^{T}$, where $T$ is the vertex set of a simplex in $L$ and where $\mathbb{R}^{T}$ is the subspace spanned by $\left\{e_{s}\right\}_{s \in T}$. The vertex set of $X$ is $\{ \pm 1\}^{S}$ and the link of each such vertex is naturally identified with $L$.

\section{Reflection groups.}

Suppose that $(W, S)$ is a Coxeter system, that $X$ is a space and that $\left(X_{s}\right)_{s \in S}$ is a family of closed subspaces. For each $x \in X$, let $S(x)=\left\{s \in S \mid x \in X_{s}\right\}$. Define an equivalence relation $\sim$ on $W \times X$ by: $(w, x) \sim\left(w^{\prime}, x^{\prime}\right)$ if and only if $x=x^{\prime}$ and $w^{-1} w^{\prime} \in W_{S(x)}$. Let $\mathcal{U}(W, X)$ denote the quotient space $(W \times X) / \sim$. The group $W$ acts on $\mathcal{U}(W, X)$; the orbit space is $X$. Moreover, we can identify $X$ with the image of $1 \times X$ in $\mathcal{U}(W, X)$. Thus, $X$ is a fundamental domain for the $W$-action. Any translate $w X$ of $X$ is called a chamber; thus, $\mathcal{U}(W, X)$ is decomposed into chambers. Each $s$ in $S$ acts on $\mathcal{U}(W, X)$ as a "reflection" in the following sense: the fixed point set of $s$ separates $\mathcal{U}(W, X)$ into two "half-spaces" which are interchanged by $s$. (A more detailed discussion and further properties of this construction can be found in $[\mathrm{V}]$ or in [D1].)

If $Y$ is a space with an action of a Coxeter group $W$, then $W$ is called a reflection group on $Y$ if $Y$ is equivariantly homeomorphic to $\mathcal{U}(W, X)$ for some subspace $X$ 
Example 10.1. Suppose that $X$ is the cone on $S$ and that $X_{s}$ denotes the point $s$. Then $\mathcal{U}(W, X)$ is the Cayley graph of $(W, S)$.

Example 10.2. Suppose that $X$ is the geometric realization of the poset $\mathcal{S}^{f}$ of $\S 7$. Thus, a $k$-simplex in $X$ corresponds to a chain $T_{0}<T_{1}<\cdots<T_{k}$, where $T_{i} \in \mathcal{S}^{f}$. For each $s$ in $S$, let $X_{s}$ denote the subcomplex consisting of those simplices such that $T_{0}=\{s\}$. Then $\mathcal{U}(W, X)$ can be identified with the barycentric subdivision of the geometric realization $\Sigma(W, S)$ of the poset of cosets $W \mathcal{S}^{f}$.

Here is what is going on in the above example. Given any poset $\mathcal{P}$, let $|\mathcal{P}|$ denote the geometric realization of its derived complex. For each $p \in \mathcal{P}$ define subposets,

$$
\begin{aligned}
& \mathcal{P}_{\leq p}=\{q \in \mathcal{P} \mid q \leq p\} \text { and } \\
& \mathcal{P}_{\geq p}=\{q \in \mathcal{P} \mid q \geq p\}
\end{aligned}
$$

Call the subcomplexes $\left|\mathcal{P}_{\leq p}\right|$ faces and the $\left|\mathcal{P}_{\geq q}\right|$ cofaces. So we have two different decompositions of $|\mathcal{P}|$, into faces or into cofaces. In the case at hand, $\Sigma(W, S)=$ $\left|W \mathcal{S}^{f}\right|$, its faces are Coxeter cells and its cofaces are intersections of chambers.

\section{$\S 11$. Applications and examples.}

a) Two dimensional complexes. Let $L$ be a finite graph and $m$ an integer $\geq 2$. Let $k$ be the girth of $L$ (the length of the shortest circuit). If $m=2$, then we assume $k \geq 4$. Let $S=\operatorname{Vert}(L)$ (the vertex set of $L$ ) and define a Coxeter matrix by

$$
m_{s s^{\prime}}= \begin{cases}1 & ; \text { if } s=s^{\prime} \\ m & ; \text { if }\left\{s, s^{\prime}\right\} \text { spans an edge } \\ \infty & ; \text { otherwise. }\end{cases}
$$

Let $W$ be the resulting Coxeter group. Our assumption implies that $N(W, S)=L$. Thus, $\Sigma(W, S)$ is a $C A T(0), P E$ 2-complex such that each 2-cell is a regular $2 m$-gon and such that the link of each vertex is $L$. Here the condition that $L$ was $C A T(1)$ was just that $k(\pi-\pi / m) \geq 2 \pi$ (which holds provided $k \geq 4$ if $m=2$ ). We can give $\Sigma$ a piecewise hyperbolic (abbreviated $P H$ ) structure by declaring each 2-cell to be a small regular $2 m$-gon in $\mathbb{H}^{2}$. Since the angles of such a $2 m$-gon will be slightly less than in the Euclidean case, we will be able to do this so that links are $C A T(1)$ provided that $k(\pi-\pi / m)>2 \pi$. This holds provided $k>4$ if $m=2$ and $k>3$ if $m=3$. Thus, provided the condition holds, $\Sigma$ can be given a $P H$ structure which is $C A T(-1)$.

These "regular" 2-complexes can be thought of as generalizations of well known examples of regular tessellations of $\mathbb{E}^{2}$ and $\mathbb{H}^{2}$.

Nadia Benakli has made a detailed study of these 2-complexes in her thesis [Be]. For example, she shows that the ideal boundary $\Sigma(\infty)$ is usually a Menger curve. Benakli also has another construction of such 2-complexes where the 2-cells are $n$-gons, with $n$ odd, provided that there is a group $G$ of automorphisms of $L$ such that $L^{\prime} / G$ is an interval ( $L^{\prime}$ is the barycentric subdivision).

b) Word hyperbolic Coxeter groups. In [M] Moussong also analyzed when the idea of the previous subsection (of replacing the Euclidean Coxeter cells) of $\Sigma$ by hyperbolic Coxeter cells works in higher dimensions. 
(*) For any subset $T$ of $S$ neither of the following holds:

(1) $W_{T}=W_{T_{1}} \times W_{T_{2}}$ with both factors infinite,

(2) $W_{T}$ is a Euclidean Coxeter group with $\operatorname{Card}(T) \geq 3$.

Here a "Euclidean Coxeter group" means the Coxeter group of an orthogonal affine reflection group on $\mathbb{E}^{n}$ with compact quotient. The "Coxeter diagrams" of these groups are listed in [B, p. 199].

Theorem 11.1. (Moussong) The following conditions are equivalent.

(i) $(W, S)$ satisfies $(*)$

(ii) $\Sigma$ can be given a PH,CAT(-1) structure,

(iii) $W$ is word hyperbolic,

(iv) $W$ does not contain a subgroup isomorphic to $\mathbb{Z}+\mathbb{Z}$.

To show (i) $\Rightarrow$ (ii) one wants to replace the cells of $\Sigma$ by Coxeter cells in $\mathbb{H}^{n}$. In order for the links to remain $C A T(1)$, one needs to know that the length of every closed geodesic in $N(W, S)$ is strictly greater than $2 \pi$ and that the same condition holds for the link of each simplex in $N(W, S)$. In his proof of Lemma 3.16, Moussong analyzed exactly when a metric flag complex has closed geodesics of length equal to $2 \pi$. In the case at hand, it was only when conditions (1) and (2) of $(*)$ hold. The implications (ii) $\Rightarrow$ (iii) $\Rightarrow$ (iv) $\Rightarrow$ (i) are all either well-known or obvious.

c) Buildings. As we mentioned in $\S 3 \mathrm{~d}$ ) associated to each building $B$ there is a Coxeter system $(W, S)$ so that each "apartment" is isomorphic to a complex associated to $(W, S)$. Traditionally, this complex is the Coxeter complex (where each chamber is a simplex). For general Coxeter groups, however, it seems more appropriate to use the complex $\Sigma^{\prime}$. Here $\Sigma^{\prime}$ denotes the barycentric subdivision of $\Sigma$. (In [CD5] we call this the "modified Coxeter complex"). This point is made by Ronan in [R, p. 184]. The buildings which arise in nature (in algebra or geometry) are usually of spherical or Euclidean type. This means that the Coxeter group $W$ is either finite or Euclidean. In the case where $W$ is Euclidean and irreducible our definition agrees with the traditional one.

In the general case let us agree that the correct definition of a building should be as a simplicial complex such that each apartment is isomorphic to $\Sigma^{\prime}$. Since, by Theorem 7.8, $\Sigma^{\prime}$ can be given the structure of a $C A T(0), P E$ cell complex, we get an induced $P E$ structure on the building $B$. As explained in $\S 3 \mathrm{c})$, the axioms for buildings imply this structure is $C A T(0)$. Thus, we have the following result, the details of which can be found in [D4].

Theorem 11.2. Any building (correctly defined) has the structure of a PE simplicial complex which is $C A T(0)$.

d) When is $\Sigma$ a manifold ? A homology manifold? Since the link of every vertex in $\Sigma$ is isomorphic to $N$, these questions can be answered as follows.

\section{Proposition 11.3.}

(i) $\Sigma$ is a homology $n$-manifold if and only if $N$ is a homology $(n-1)$-manifold with the homology of $S^{n-1}$.

(ii) For $n \geq 5, \Sigma$ is a topological n-manifold if and only if $N$ is as in (i) and $N$ is simply connected. 
Statements (i) and (iii) are just restatements of the definitions and statement (ii) follows from Edwards' Theorem 5.10.

We begin by discussing some examples of (iii) when $N$ is a $P L$ triangulation of $S^{n-1}$.

Example 11.4. (Lanner $[\mathrm{L}])$. Suppose that $N(W, S)$ is isomorphic to the boundary complex of an $n$-simplex. Then $n=\operatorname{Card}(S)-1, W$ is infinite, and for every proper subset $T$ of $S$, the group $W_{T}$ is finite. Such groups were classified in 1950 by Lanner: they are either irreducible Euclidean reflection groups or hyperbolic reflection groups. In both cases a fundamental chamber is an $n$-simplex. In the Euclidean case there are four families in each dimension $n$ and a five exceptional cases in dimensions $\leq 8$. In the hyperbolic case, in dimension 2 , we have the hyperbolic triangle groups: these are the groups such that $\operatorname{Card}(S)=3$ and the 3 entries $p, q, r$ of the Coxeter matrix above the diagonal satisfy $(1 / p)+(1 / q)+$ $(1 / r)<1$. Furthermore, there are 9 hyperbolic examples in dimension 3,5 more in dimension 4 , and none in dimensions $>4$. Complete lists can be found on pages 133 and 199 of $[\mathrm{B}]$.

Example 11.5. (Andreev [A]) Suppose that $N$ is a triangulation of $S^{2}$ and that condition (*) of subsection b) holds. Then Andreev proved that $W$ can be realized (uniquely, up to conjugation by an isometry) as a reflection group on $\mathbb{H}^{3}$. In fact, he shows that there is a convex cell $C^{3}$ in $\mathbb{H}^{3}$, the polar dual of which is $N$. (Thus, the faces of $C^{3}$ corresponding to $s$ and $s^{\prime}$ make a dihedral angle of $\pi / m_{s s^{\prime}}$.) $W$ is the group generated by reflections across the faces of $C^{3}$.

Example 11.6. ([T], [D2] and [CD2]). Suppose $L$ is the boundary complex of a $n$-dimensional octahedron (i.e., $L$ is the $n$-fold join of $S^{0}$ with itself). Let $W^{\prime}$ be a finite Coxeter group of rank $n$. Use $W^{\prime}$ to label the edges of one $(n-1)$-simplex in $L$. Label the other edges 2. This defines a Coxeter group $W$, with $N(W, S)=L$. Each chamber of $W$ on $\Sigma$ is combinatorially equivalent to the cone on the dual cellulation of $N$, i.e., each chamber is a combinatorial cube. If $W^{\prime}=(\mathbb{Z} / 2)^{n}$, then each chamber actually is an $n$-cube.

In the case where $W^{\prime}=S_{n+1}$, the symmetric group, the $W$-manifolds $\Sigma$ actually arise in nature. For example, there is an obvious homomorphism $W \rightarrow W^{\prime} \times(\mathbb{Z} / 2)^{n}$ with kernel $\Gamma_{0}$. Tomei showed in $[\mathrm{T}]$ that the $n$-manifold $\Sigma / \Gamma_{0}$ can be identified with the manifold of all tridiagonal, symmetric $(n+1) \times(n+1)$ matrices with constant spectrum (of distinct eigenvalues). This is also explained in [D2]. From a completely different direction, it is shown in [CD2] that for a certain torsionfree, finite-index, normal subgroup $\Gamma_{1}$ of $W, \Sigma / \Gamma_{1}$ can be identified with Gromov's "Moebius band" hyperbolization construction applied to boundary of a $(n+1)$-cube, and that for a different subgroup $\Gamma_{2}, \Sigma / \Gamma_{2}$ can be identified with the "product with interval" hyperbolization construction applied to the boundary of a simple regular $(n+1)$-cell.

Example 11.7. Let $L$ be any flag complex which is a $P L$ triangulation of $S^{n-1}$, and let $(W, S)$ be the corresponding right-angled Coxeter system with $N=L$.

Proposition 11.8. Hopf's Conjecture (from $\S 6$ ) for PE cubical manifolds is equivalent to Conjecture 6.6.

Proof. We saw in $\S 6$ that Conjecture 6.6 implies Hopf's Conjecture for PE cubical 
$X^{2 n}$ be the manifold constructed in Corollary 9.6. By the Combinatorial GaussBonnet Theorem (Theorem 6.3),

$$
\begin{aligned}
\chi\left(X^{2 n}\right) & =\sum P(L) \\
& =[W: \Gamma] P(L) .
\end{aligned}
$$

Hence, $\chi\left(X^{2 n}\right)$ and $P(L)$ have the same sign.

Example 11.9. Suppose that $R$ is a ring and that $L$ is a flag complex which is a $R$-homology $(n-1)$-manifold with the same homology (over $R$ ) as $S^{n-1}$ and let $(W, S)$ be the right-angled Coxeter system with $N=L$. For example, we could take $R=\mathbb{Z}\left[\frac{1}{m}\right]$ and $L$ to be the lens space $S^{2 k-1} /(\mathbb{Z} / m)$ or the suspension of such a lens space. Then $\Sigma$ is an $R$-homology $n$-manifold. Moreover, the ideal boundary $\Sigma(\infty)$ has the same homology (over $R$ ) as does $S^{n-1}$. (Compare Theorems 5.8 and 5.10.) It follows that $W$ is a virtual Poincaré duality group over $R$ in the sense that any torsion-free subgroup $\Gamma$ of finite index in $W$ satisfies Poincaré duality over $R$. However, as is shown in [D5] or in [DL], if $L$ is not an integral homology $(n-1)$ sphere, then neither is $\Sigma(\infty)$. Hence, for such an $L, W$ is not a virtual Poincaré duality group over $\mathbb{Z}$.

e) Cohomological dimension. The cohomological dimension of a torsion-free group $\Gamma$ over a ring $R$, denoted by $c d_{R}(\Gamma)$, can be defined as the smallest dimension of an $R$-acyclic $C W$ complex on which $\Gamma$ can act freely. (If the $\operatorname{ring} R$ is $\mathbb{Z}$, then we omit it from our notation.) If a group $G$ is not torsion-free, but contains a finiteindex, torsion-free subgroup $\Gamma$, then the virtual cohomological dimension of $G$, denoted $v c d_{R}(G)$, is defined by $v c d_{R}(G)=c d_{R}(\Gamma)$. If $\Gamma$ acts freely and cocompactly on a contractible complex $\Omega$, then $c d_{R}(\Gamma)=\sup \left\{n \mid H_{c}^{n}(\Omega ; R) \neq 0\right\}$. (See p. 209 in $[\mathrm{Br} 1]$.

Example 11.10. (Bestvina-Mess $[\mathrm{BM}]$ ). Suppose that $L$ is a flag complex homeomorphic to $\mathbb{R} P^{2}$, that $(W, S)$ is the right-angled Coxeter system with $N=L$, and that $\Sigma=\Sigma(W, S)$. By Theorem 5.10, $\Sigma(\infty)$ is an inverse limit of connected sums of an increasing number of projective planes. In other words, $\Sigma(\infty)$ is an inverse limit of nonorientable surfaces of increasing genus. Thus, $H^{n}(\Sigma(\infty) ; \mathbb{Q})=0$ for $n \geq 2$, while $H^{2}(\Sigma(\infty) ; \mathbb{Z})=\mathbb{Z} / 2$. Consequently, $H_{c}^{n}(\Sigma ; \mathbb{Q})=0$ for $n \geq 3$, and $H_{c}^{3}(\Sigma ; \mathbb{Z})=\mathbb{Z} / 2$. It follows that $\operatorname{vcd}_{\mathbb{Q}}(W)=2$ and $\operatorname{vcd}(W)=3$.

Example 11.11. (Dranishnikov [Dr] and Dicks-Leary [DL]) There are similar examples using other Moore spaces than $\mathbb{R} P^{2}$. For example, suppose a flag complex $L_{2}$ is 2 -dimensional and has $H_{1}\left(L_{2} ; \mathbb{Z}\right)=\mathbb{Z} / 3$ and $\bar{H}_{i}\left(L_{2} ; \mathbb{Z}\right)=0$, for $i \neq 1$. Let $\left(W_{2}, S_{2}\right)$ be the corresponding Coxeter system. Let $\left(W_{1}, S_{1}\right)$ be the Coxeter system of the previous example. Put $\Sigma_{1}=\Sigma\left(W_{1}, S_{1}\right), \Sigma_{2}=\Sigma\left(W_{2}, S_{2}\right)$ and $\Sigma=\Sigma_{1} \times \Sigma_{2}$. So, $\operatorname{dim} \Sigma=6$. The Kunneth formula implies that $H_{c}^{6}(\Sigma ; \mathbb{Z})=\mathbb{Z} / 2 \otimes \mathbb{Z} / 3=0$. Thus, $\operatorname{vcd}\left(W_{1}\right)=\operatorname{vcd}\left(W_{2}\right)=3$, while $\operatorname{vcd}\left(W_{1} \times W_{2}\right) \leq 5$. Hence, cohomological dimension is not additive for direct products.

Since $W$ acts on the contractible complex $\Sigma$ with finite isotropy groups, we always have that $\operatorname{vcd}(W) \leq \operatorname{dim} \Sigma$. As we shall see below, the inequality can be strict.

Let $X$ be a $C W$ complex and $\left(X_{s}\right)_{s \in S}$ a family of subcomplexes as in $\S 10$. For any subset $T$ of $S$ put

$$
X_{T}=\bigcap X_{s}
$$


and $X_{\emptyset}=X$. Suppose that the following two conditions hold:

(i) $X_{T}=\emptyset, T \notin \mathcal{S}^{f}$

(ii) $X_{T}$ is acyclic, $T \in \mathcal{S}^{f}$.

It then follows from Theorem 10.1 in $[\mathrm{D} 1]$ that $\mathcal{U}(W, X)$ is acyclic and, by construction, that $W$ acts on $\mathcal{U}(W, X)$ with finite isotropy groups. Hence, $v c d(W) \leq \operatorname{dim} X$. In [Bes1] Bestvina shows that, in fact, $\operatorname{vcd}(W)$ is equal to the smallest possible dimension of such an $X$. In [Dr] Dranishnikov gives an explicit construction of such a minimal $X$. (Of course, in most cases, for example if $N(W, S)$ carries some top-dimensional homology, then $\operatorname{vcd}(W)=\operatorname{dim} \Sigma$.)

f) The Eilenberg-Ganea Problem. The geometric dimension of a torsion-free group $\Gamma$, denotes $g d(\Gamma)$, is the smallest dimension of a $K(\Gamma, 1)$ complex. Equivalently, it is the smallest dimension of a contractible $C W$ complex on which $\Gamma$ can act freely. The following result is proved in $[\mathrm{EG}]$ when $c d(\Gamma) \neq 1$ and in [Sta2] in the case $c d(\Gamma)=1$.

Theorem 11.12. (Eilenberg-Ganea, Stallings)

(i) If $c d(\Gamma) \neq 2$, then $c d(\Gamma)=\operatorname{gd}(\Gamma)$.

(ii) If $\operatorname{cd}(\Gamma)=2$, then either $\operatorname{gd}(\Gamma)=2$ or $\operatorname{gd}(\Gamma)=3$.

The Eilenberg-Ganea Problem is the question of whether or not there exists a group $\Gamma$ with $c d(\Gamma)=2$ and $g d(\Gamma)=3$.

Let $L$ be 2-dimensional flag complex which is (a) acyclic and (b) not simply connected. Let $(W, S)$ be the corresponding right-angled Coxeter system with $N=$ $L$. If $X$ denotes the geometric realization of $\mathcal{S}^{f}$ then, as in Example $10.2, \mathcal{U}(W, X)=$ $\Sigma$. Let $X_{0}$ be the geometric realization of $\mathcal{S}_{>\emptyset}^{f}$ (so that $X_{0}$ is the barycentric subdvision of $L$ and $X$ is the cone on $X)$ and put $X_{0 s}=X_{s}$ and $\Sigma_{0}=\mathcal{U}\left(W, X_{0}\right)$. By the last paragraph of subsection e), $\Sigma_{0}$ is an acyclic 2-complex. It follows that for any torsion-free subgroup $\Gamma$ of finite index in $W, c d(\Gamma)=2$. On the other hand, the only natural contractible complex on which $\Gamma$ acts is $\Sigma$, which has dimension 3. This leads to the following

Conjecture 11.13. (Bestvina) For any $\Gamma$ as above, $g d(\Gamma)=3$.

The reason for believing this is that it seems that $X_{0}$ should embed in the universal cover $E \Gamma$ of any $K(\Gamma, 1)$. Furthermore, since $\pi_{1}\left(X_{0}\right)$ is not trivial it should be impossible to embed it in any contractible 2-complex, since it should not be possible to kill $\pi_{1}\left(X_{0}\right)$ by adding the same number of 1- and 2-cells. (This is related to the Kervaire Conjecture: if $G$ is a nontrivial group, then any group obtained from $G$ by adding one generator and one relation is also nontrivial.)

\section{g) Is $\Sigma$ homeomorphic to $\mathbb{R}^{n}$ ?}

Proposition 11.14. (i) If $N$ is a PL triangulation of $S^{n-1}$, then $\Sigma$ is $P L$ homeomorphic to $\mathbb{R}^{n}$.

(ii) If $N$ is a PL homology sphere (i.e., $N$ is a PL manifold) and $\pi_{1}(N)$ is not trivial, then $\Sigma$ is not simply connected at infinity. (However, $\Sigma$ is not a manifold, rather it is only a homology manifold.)

(iii) If $N$ is a simply connected homology manifold with the homology of $S^{n-1}$, then, for $n \geq 5, \Sigma$ is a contractible manifold.

Proof. Statement (i) follows from Stone's Theorem 5.5, statement (ii) from Propo- 
We are now in position to tackle Questions 5.3 and 5.4.

Proposition 11.15. ([DJ, p. 383]) For each $n \geq 5$, there is a Coxeter system $(W, S)$ so that the corresponding $P E$ polyhedron $\Sigma$ is

(a) $C A T(0)$

(b) a topological n-manifold, and

(c) not homeomorphic to $\mathbb{R}^{n}$.

Proof. Let $A^{n-1}$ be a compact acyclic $P L$ manifold with boundary such that (1) $\pi_{1}(\partial A) \rightarrow \pi_{1}(A)$ is onto and (2) the double of $A$ along $\partial A$ is not simply connected. Take a triangulation of $A$ as a flag complex so that $\partial A$ is a full subcomplex. Let $N$ be the simplicial complex resulting from attaching the cone on $\partial A$ to $A$. Let $(W, S)$ be the right-angled Coxeter system with $N(W, S)=N$. By (1), $N$ is simply connected, so by Proposiition 11.14 (iii), $\Sigma$ is a topological $n$-manifold. Let $\left(W_{1}, S_{1}\right)$ be the right-angled Coxeter system whose nerve is the double of $A$ along $\partial A$. It follows from (2) and part (ii) of Proposition 11.14 that the resulting contractible complex is not simply connected at infinity. On the other hand, $W_{1}$ can be identified with an index two subgroup of $W$. (Double the fundamental chamber $X$ of $\Sigma$ along $X_{s}$ where $s$ corresponds to the cone point in $N$.) Since the fundamental group at infinity of $\Sigma$ depends only on $W$ (or $W_{1}$ ) we see that $\Sigma$ is also not simply connected at infinity and hence, not homeomorphic to $\mathbb{R}^{n}$.

In $[\mathrm{ADG}]$ it is proved that in the case where $N(W, S)$ is a nonsimply connected $P L$ homology sphere (as in Proposition 11.14 (ii)), a modified version of $\Sigma$ can be taken to be a topological manifold. The idea is similar to that in the previous proof: one blows up the $P L$ singularities of $\Sigma$ from isolated vertices into intervals. More precisely, we have the following result.

Proposition 11.16. ([ADG]) Given a PL homology sphere $L^{n-1}, n \geq 5$, there exists a right-angled Coxeter system $(W, S)$, with $N(W, S)=L$, and a PE cubical complex $\Sigma_{1}$ with $W$ - action such that (a) $\Sigma_{1}$ is $C A T(0)$ and (b) $\Sigma_{1}$ is a topological $n$-manifold.

Idea of Proof. We can find a codimension-one homology sphere $L_{0} \subset L$ such that 1) $L_{0}$ divides $L$ into two pieces $L_{1}$ and $L_{2}$ (each of which is an acyclic manifold with boundary) and 2) $\pi_{1}\left(L_{0}\right) \rightarrow \pi_{1}\left(L_{i}\right)$ is onto, for $i=1,2$. Triangulate $L$ as a flag complex so that $L_{0}$ is a full subcomplex and let $(W, S)$ be the right-angled Coxeter system such that $N(W, S)=L$. For $i=1,2$, let $N_{i}$ denote the union of $L_{i}$ with the cone over $L_{0}$. Then $N_{i}$ is simply connected. In the construction of $\Sigma$ a fundamental chamber is essentially a cubical cone over $L$. As explained in [ADG], to construct $\Sigma_{1}$, one uses as a chamber the union of two cubical cones: one over $N_{1}$ and the other over $N_{2}$. These cones are glued together along the cone on $L_{0}$. Such a chamber now has $P L$ singularities along an interval which connects the two cone points $c_{1}$ and $c_{2}$. The link of $c_{i}$ is $N_{i}$, which is simply connected; hence, by Edwards' Theorem 5.11, $\Sigma_{1}$ is a topological manifold.

Remark 11.17. In the previous two propositions we have shown that Questions 5.3 a) and 5.4 a) have negative answers. As for part b), let $\Sigma_{1}$ be as in the previous proposition and consider the CAT (0) manifold $X=\Sigma_{1} \times \mathbb{R}$. The group $W \times \mathbb{Z}$ acts on $X$ with compact quotient. Since $X$ is simply connected at infinity, it is homeomorphic to $\mathbb{R}^{n+1}$ (by $\left[\right.$ Sta1]). On the other hand, $X(\infty)$ is the join of $\Sigma_{1}(\infty)$ 
boundary is a manifold, then must it be a sphere? It is proved in $[A G]$ that the answer is no. The construction is very similar to the one in the above proof of Proposition 11.16. Indeed, if $N_{1}$ and $N_{2}$ are as in the above proof, then one takes two Euclidean cones (or two hyperbolic cones) on $S_{1}$ and $N_{2}$ and glue them together along the cone on $L_{0}$. The resulting space $Y^{n}$ is a complete CAT (0) manifold (it is CAT $(-1)$ if we use hyperbolic cones) and $Y(\infty)$ is the original $P L$ homology sphere $L^{n-1}$. On the other hand, it seems unlikely (because of $[\mathrm{Wr}]$ ) that one could construct such an example with a cocompact group of isometries. Thus, the answer to Question 5.4 c) is probably "yes". 


\section{Artin Groups.}

As explained in $\S 12$ any Coxeter group (finite or not) has a representation as a reflection group on a real vector space. Take the complexification of this vector space. It contains a certain convex open subset such that after deleting the reflection hyperplanes, we obtain an open manifold $M$ on which the Coxeter group $W$ acts properly and freely. The fundamental group of $M / W$ is the "Artin group" $A$ associated to $W$. When $W$ is finite, Deligne proved that $M / W$ is a $K(A, 1)$ space. The conjecture that this should always be the case, is here called the "Main Conjecture". The purpose of this chapter is to outline some work on this conjecture in [CD5] and [CD6].

Associated to the Artin group there is a cell complex $\Phi$ (which is very similar to $\Sigma$ ). It turns out (Corollary 15.2) that proving the Main Conjecture for $W$ is equivalent to showing $\Phi$ is contractible. The complex $\Phi$ has a natural $P E$ structure, which we conjecture is always $C A T(0)$. We do not know how to prove this; however, in $\S 16$ we show that there is a (less natural) cubical structure on $\Phi$ and that in "most cases" it is $C A T(0)$. Hence, the Main Conjecture holds in most cases.

\section{$\S 12$. Hyperplane Complements.}

Let $S_{n}$ denote the symmetric group on $n$ letters. It acts on $\mathbb{R}^{n}$ by permutation of coordinates. In fact, this action is as an orthogonal reflection group: the reflections are the transpositions $(i j), 1 \leq i<j \leq n$, the corresponding reflection hyperplanes are the $H_{i j}=\left\{x \in \mathbb{R}^{n} \mid x_{i}=x_{j}\right\}$. Complexifiying we get an action of $S_{n}$ on $\mathbb{C}^{n}=\mathbb{R}^{n} \otimes \mathbb{C}$ such that $S_{n}$ acts freely on

$$
M=\mathbb{C}^{n}-\bigcup\left(H_{i j} \otimes \mathbb{C}\right) .
$$

Thus, $M / S_{n}$ is the configuration space of unordered sets of $n$ distinct points in $\mathbb{C}$.

It is a classical fact that the fundamental group of $M / S_{n}$ is $B_{n}$, the braid group on $n$ strands. The following result is also classical.

Theorem 12.1. (Fox-Neuwirth $[\mathrm{FN}]$ )

1) $M$ is a $K(\pi, 1)$ space, where $\pi$ is $P B_{n}$ the pure braid group (i.e., $P B_{n}$ is the kernel of $\left.B_{n} \rightarrow S_{n}\right)$.

2) $M / S_{n}$ is a $K(\pi, 1)$ space, for $\pi=B_{n}$.

Next suppose that $W$ is a finite reflection group on $\mathbb{R}^{n}$ and that

$$
M=\mathbb{C}^{n}-\bigcup_{r} H_{r} \otimes \mathbb{C}
$$

where the union is over all reflections $r$ in $W$ (i.e., all conjugates of elements in $S$ ) and where $H_{r}$ is the hyperplane fixed by $r$. Arnold and Brieskorn asked if the analogous result to Theorem 12.1 holds in this context. In [De], Deligne proved that this was indeed the case.

Theorem 12.2. (Deligne [De]) Suppose that $W$ is a finite reflection group. Then $M / W$ is a $K(\pi, 1)$ space, where $\pi$ is the "Artin group" associated to $W$ (as defined below).

Artin groups. Suppose that $(W, S)$ is a Coxeter system and that $M=\left(m_{s s^{\prime}}\right)$ is the associated Coxeter matrix. Introduce a new set of symbols $X=\left\{x_{s} \mid s \in S\right\}$ one for each element of $s$.

Notation. If $m$ is an integer $\geq 2$, then let $\operatorname{prod}(x, y ; m)$ denote the word: $x y x \cdots$, 
Definition 12.3. The Artin group associated to $(W, S)$ (or to $M$ ) is the group generated by $X$ and with relations:

$$
\operatorname{prod}\left(x_{s}, x_{s^{\prime}} ; m_{s s^{\prime}}\right)=\operatorname{prod}\left(x_{s^{\prime}}, x_{s} ; m_{s s^{\prime}}\right)
$$

where $\left(s s^{\prime}\right)$ range over all elements of $S \times S$ such that $s \neq s^{\prime}$ and $m_{s s^{\prime}} \neq \infty$.

Remark 12.4. If we add the relations $\left(x_{s}\right)^{2}=1$, then the relation appearing in the previous definition can be rewritten as $\left(x_{s} x_{s^{\prime}}\right)^{m_{s s^{\prime}}}=1$; hence, we recover the standard presentation of $W$. Thus, if $A$ is the Artin group associated to $(W, S)$, we see that there is a canonical surjection $p: A \rightarrow W$ which send $x_{s}$ to $s$.

Example 12.5. If $W$ is $S_{n}$, then the associated Artin group is $B_{n}$.

It is natural to ask if Theorem 12.2 holds in the case where $W$ is infinite. In order to make sense of this question we first need to discuss what is meant by a "linear reflection group" in the infinite case.

Linear reflection groups. Let $V$ be a finite dimensional real vector space. A linear reflection on $V$ means a linear involution with fixed space a hyperplane.

Suppose that $C$ is a convex polyhedral cone in $V$ (Figure 5 ) and that $S$ is a finite set which indexes the set of codimension-one faces of $C$. Thus, $\left(C_{s}\right)_{s \in S}$ will be the family of codimension-one faces of $C$. Let $H_{s}$ denote the linear hyperplane spanned by $C_{s}$.

Figure 5

For each $s \in S$, choose a reflection $\rho_{s}$ with fixed subspace $H_{s}$. Let $W$ denote the subgroup of $G L(V)$ generated by $\left\{\rho_{s} \mid s \in S\right\}$.

Definition 12.6. $W$ is a linear reflection group if $w \stackrel{\circ}{C} \cap \stackrel{\circ}{C}=\emptyset$ for all $w \in W, w \neq 1$. (Here $\stackrel{\circ}{C}$ denotes the interior of $C$.)

Definition 12.7. Let

$$
\bar{I}=\bigcup_{w \subset W} w C
$$

and let $I$ denote the interior of $\bar{I} . \bar{I}$ is called the Tits cone.

Example 12.8. Consider the quadratic form model of $\mathbb{H}^{2}$ : the hyperbolic plane is identified with one sheet of a hyperboloid in $\mathbb{R}^{2,1}$ (3-dimensional Minkowski space). An isometric reflection on $\mathbb{H}^{2}$ extends to a linear reflection on $\mathbb{R}^{2,1}$ preserving the indefinite quadratic form. Now suppose that $W$ is the reflection group on $\mathbb{H}^{2}$ generated by the reflections across the edges of a hyperbolic polygon with angles of the form $\pi / m$. Then $W$ can be regarded as a linear reflection group on $\mathbb{R}^{2,1}$. (See 
Figure 6

In this case the interior $I$ of the Tits cone is just the interior of the light cone.

Theorem 12.9. (Vinberg $[\mathrm{V}]$ ) Suppose that $W \subset G L(V)$ is a linear reflection group with fundamental polyhedral cone $C$. Put $C^{f}=\left\{x \in C \mid W_{x}\right.$ is finite $\}$. Then

(i) $(W, S)$ is a Coxeter system,

(ii) $\bar{I}$ is a convex cone,

(iii) $K$ is $W$-stable and $W$ acts properly on it,

(iv) $I \cap C=C^{f}$,

(v) the poset of faces of $C^{f}$ is $\mathcal{S}^{f}$ (where $\mathcal{S}^{f}=\left\{T \subset S \mid W_{T}\right.$ is finite $\}$.

Let $W$ be as in Vinberg's Theorem and consider the domain $V+i I$ in $V \otimes \mathbb{C}(V+i I$ denotes the set of vectors whose imaginary part lies in $I$ ). Set

$$
M=(V+i I)-\bigcup\left(H_{r} \otimes \mathbb{C}\right)
$$

The following is the main conjecture which we shall be concerned with in this chapter. According to [Lek] it is due to Arnold, Pham and Thom.

Conjecture 12.10. (the "Main Conjecture") $M / W$ is a $K(\pi, 1)$ space, where $\pi=$ $A_{W}$, the Artin group associated to $(W, S)$.

Some progress on this was made in the thesis of H. van der Lek [Lek], where the following result is proved. (Another proof can be found in [CD5].)

Proposition 12.11. (van der Lek) $\pi_{1}(M / W)=A_{W}$.

The Main Conjecture can also be formulated in terms of the cell complex $\Sigma$ which was introduced in the previous chapter in $\S 9$. In fact, in view of Theorem $12.9(\mathrm{v})$, the following lemma is not surprising.

Lemma 12.12. ([CD5, §2]) There are $W$-isovariant homotopy equivalences:

$$
\begin{gathered}
I \sim \Sigma \text { and } \\
V+i I \sim I \times I \sim \Sigma \times \Sigma .
\end{gathered}
$$

Set

$$
Y=(\Sigma \times \Sigma)-\bigcup_{r} \Sigma_{r} \times \Sigma_{r}
$$

where $\Sigma_{r}$ denotes the subcomplex of $\Sigma^{\prime}$ fixed by $r$. Then

$$
Y / W \sim M / W
$$


Conjecture 12.13. (reformulation of the Main Conjecture) $Y / W$ is a $K\left(A_{W}, 1\right)$.

\section{$\S 13$. The Salvetti complex.}

In this section, which is independent of the last three sections of this chapter, we describe a $P E$ cell complex $\tilde{\Sigma}$ homotopy equivalent to $M$. The quotient space $\tilde{\Sigma} / W$ is a finite $C W$ complex. Hence, when the Main Conjecture holds, $\tilde{\Sigma} / W$ will be a $K\left(A_{W}, 1\right)$ space. The complete details of this construction are given in [CD6].

In $\S 7$, we considered two posets:

$$
\begin{aligned}
\mathcal{S}^{f} & =\left\{T \subset S \mid W_{T} \text { is finite }\right\} \text { and } \\
W \mathcal{S}^{f} & =\coprod_{T \in \mathcal{S}^{f}} W / W_{T} .
\end{aligned}
$$

Here we consider a third poset $W \times \mathcal{S}^{f}$. The partial ordering on $W \times \mathcal{S}^{f}$ is defined as follows: $(w, T)<\left(w^{\prime}, T^{\prime}\right)$ if and only if

(i) $T<T^{\prime}$

(ii) $w^{-1} w^{\prime} \in W_{T^{\prime}}$, and

(iii) for all $t \in T, \ell\left(w^{-1} w^{\prime}\right)<\ell\left(t w^{-1} w^{\prime}\right)$, (where $\ell$ denotes word length in $W$ ).

There is a natural projection $\pi: W \times \mathcal{S}^{f} \rightarrow W \mathcal{S}^{f}$ defined by $(w, T) \rightarrow w W_{T}$. Conditions (i) and (ii) just mean that $\pi$ is order-preserving. Condition (iii) comes out of the proof of Proposition 13.1, below.

The quickest way to define $\tilde{\Sigma}$ is to first define its barycentric subdivision $\tilde{\Sigma}^{\prime}$; it is the geometric realization of the derived complex of $W \times \mathcal{S}^{f}$. One then observes that the union of simplices with maximal vertex is $(w, T)$ can be identified with a Coxeter cell of type $W_{T}$.

If $Z$ is a cell complex then $\mathcal{P}(Z)$ denotes the poset of cells in $Z$. For example, $\mathcal{P}(\Sigma)=W \mathcal{S}^{f}$.

Proposition 13.1. (Salvetti $[\mathrm{S}]$ and $[\mathrm{CD} 6]$ ). There is a $P E$ cell complex $\tilde{\Sigma}$ such that

(i) $\mathcal{P}(\tilde{\Sigma})=W \times \mathcal{S}^{f}$,

(ii) each cell of $\tilde{\Sigma}$ is a Coxeter cell,

(iii) $W$ acts freely on $\tilde{\Sigma}$,

(iv) $\tilde{\Sigma}$ is $W$-equivariantly homotopy equivalent to $M$ (or to $Y$ ) and hence, $\tilde{\Sigma} / W \sim$ $M / W$.

Sketch of Proof. First, for each $(w, T)$ in $W \times \mathcal{S}^{f}$ we will describe two open sets in $\Sigma^{\prime}$. Let $U_{(w, T)}^{\prime}$ denote the open star of the vertex corresponding to $w W_{T}$ in $\Sigma^{\prime}$. Let $U_{(w, T)}^{\prime \prime}$ denote the intersection of the open "half spaces" in $\Sigma^{\prime}$ which are bounded by the $\Sigma_{r}$ with $r$ a reflection in $w W_{T} w^{-1}$ and which contain the vertex $w \cdot\left(U_{(w, T)}^{\prime \prime}\right.$ is an open "sector".) We note that $U_{(w, T)}^{\prime \prime}$ contains no point in $U_{(w, T)}^{\prime}$ with nontrivial isotropy group.

Consider $Y=(\Sigma \times \Sigma)-\cup\left(\Sigma_{r} \times \Sigma_{r}\right)$. Let $U_{(w, T)}=U_{(w, T)}^{\prime} \times U_{(w, T)}^{\prime \prime}$. One checks easily that a) $U_{(w, T)} \subset Y$, b) $\left\{U_{(w, T)}\right\}$ is an open cover of $Y$, c) each nonempty intersection of elements in this cover is contractible, and d) the nerve of this cover is $\tilde{\Sigma}^{\prime}$ (the geometric realization of $\left.\left(W \times \mathcal{S}^{f}\right)^{\prime}\right)$. The proposition follows.

Remark. In [S] Salvetti carries out the above construction for arbitrary hyperplane complements. The special case above is done in [CD6]. When $W$ is the symmetric 
The $C W$ complex $\tilde{\Sigma} / W$ has one cell of dimension Card $(T)$ for each $T \in \mathcal{S}^{f}$. In particular, when $W$ is finite, $\tilde{\Sigma} / W$ is the $C W$ complex formed by identifying faces of a single Coxeter cell: the precise identifications can be worked out using conditions (i), (ii) and (iii) in the definition of the partial order on $W \times \mathcal{S}^{f}$.

Corollary 13.2. The Main Conjecture holds if and only if $\tilde{\Sigma} / W$ is a $K\left(A_{W}, 1\right)$ space.

Example 13.3. The Main Conjecture holds when $W$ is finite. In particular, $\tilde{\Sigma} / W$ is a $K(A, 1)$ when $A$ is a braid group. For example, when $A=B_{3}$, a $K\left(B_{3}, 1\right)$ can be constructed by identifying edges of a hexagon in the following pattern:

\section{Figure 7}

Example 13.4. If $W=(\mathbb{Z} / 2)^{n}$, then $\widetilde{\Sigma} / W$ can be identified with the $n$-torus $T^{n}$ with its usual cell structure (obtained by identifying opposite faces of the $n$-cube).

Example 13.5. Suppose $W$ is right-angled. The Main conjecture holds for $W$ (see Theorem 16.2 below). In this case $\widetilde{\Sigma} / W$ can be identified with a subcomplex of $T^{n}$, $n=$ Card $(S)$, as follows. Let $\left(z_{s}\right)_{s \in S}$ be the standard linear coordinates on $T^{n}$. Given a point $z=\left(z_{s}\right)_{s \in S}$ in $T^{n}$ define the support of $z$ by $\operatorname{Supp}(z)=\left\{s \in S \mid z_{s} \not \equiv 0\right.$ $(\bmod 1)\}$. Then $\widetilde{\Sigma} / W$ can be identified with the subcomplex of $T^{n}$ consisting of all $z \in T^{n}$ such that $\operatorname{Supp}(z)$ is the vertex set of a simplex in $N(W, S)$. Thus, $\widetilde{\Sigma} / W$ is a union of subtori, one for each simplex in $N(W, S)$.

In [BB], Bestvina and Brady made use of such "right-angled Artin groups" to construct some startling examples of non-finitely presented groups. Suppose that $N(W, S)$ is a finite acyclic complex which is not simply connected (as in $11 \mathrm{f}$ )). Let $A$ be the associated Artin group and $\varphi: A \rightarrow \mathbb{Z}$ the homomorphism which sends each generator $x_{s}$ to a generator of $\mathbb{Z}$ and let $H$ denote the kernel of $\varphi$. Let $f: \widetilde{\Sigma} / W \rightarrow S^{1}$ be the restriction of the linear map $T^{n} \rightarrow S^{1}$ which sends $z$ to $\Sigma z_{s}$ $(\bmod 1)$. Then $f$ induces $\varphi$ on $\pi_{1}$. It is proved in [BB] that (a) $H$ is not finitely presented and (b) for any $\theta \in S^{1}$, the inverse image of $f^{-1}(\theta)$ in the universal cover of $\widetilde{\Sigma} / W$ is acyclic. Such $H$ were the first examples of torsion-free groups which were not finitely presentable and could act freely and cocompactly on acyclic complexes. Note that $c d(H)=\operatorname{dim}\left(f^{-1}(\theta)\right)=\operatorname{dim}(N(W, S))$, while the dimension of the universal cover of $\widetilde{\Sigma}$ is one greater. So, when $N(W, S)$ is two dimensional, $H$ is a good candidate for a counterexample to the Eilenberg-Ganea Problem. In fact, Bestvina and Brady proved that when $N(W, S)$ is the 2-skeleton of Poincaré's homology 3-sphere, then either $H$ is such a counterexample or else Whitehead's Conjecture (that every subcomplex of an aspherical 2-complex is aspherical) is false. 
Corollary 13.6. ([CD6]) Suppose the Main Conjecture holds for $(W, S)$. Then

(i) $\operatorname{cd}\left(A_{W}\right)=\operatorname{dim} \tilde{\Sigma}=\operatorname{dim} \Sigma$.

(ii) $\chi\left(A_{W}\right)=\chi(\tilde{\Sigma} / W)=1-\chi(N(W, S))$.

A naive idea for proving the Main Conjecture would be to show that $K(\tilde{\Sigma}) \leq 0$. This actually works when $W$ is right-angled (as is proved in [CD6]); moreover, this fact plays a key role in the work of Bestvina and Brady. However, it does not work in general. For example, when $A=B_{3}$, the link of a vertex in the complex in Example 13.3 is the following graph.

Figure 8

Each edge length is $2 \pi / 3$, but then the digons have length $4 \pi / 3$ which is $<2 \pi$.

\section{$\S 14$. Complexes of groups.}

\section{Graphs of groups.}

We begin by recalling some well-known results from the theory of graphs of groups (cf. [Se]).

Let $\Omega$ be a graph, $\mathcal{P}(\Omega)$ the poset of cells in $\Omega$ and $\mathcal{P}(\Omega)^{o p}$ the dual poset, thought of as a category. A graph of groups over $\Omega$ is a functor $\mathcal{G}$ from $\mathcal{P}(\Omega)^{\text {op }}$ to the category of groups and monomorphisms. Thus, to each vertex $v$ of $\Omega$ we are given a group $\mathcal{G}(v)$ and similarly a group $\mathcal{G}(e)$ for each edge $e$. Moreover, if $v$ is a vertex of $e$, then there is a monomorphism $\mathcal{G}(e) \rightarrow \mathcal{G}(v)$.

From these data one can construct a group $G$, called the fundamental group of $\mathcal{G}$ and denoted by $\pi_{1}(\mathcal{G})$. The basic result in the theory is the following.

Theorem 14.1. ([Se]). Given a graph of groups $\mathcal{G}$ over $\Omega$, there exists a tree $T$ with $G$-action $\left(G=\pi_{1}(\mathcal{G})\right)$ so that the following hold.

(i) $T / G=\Omega$

(ii) Suppose $e$ is an edge of $\Omega, v$ a vertex of $e, \tilde{e}$ a lift of $e$ to $T$ and $\tilde{v}$ the corresponding vertex of $\tilde{e}$. Then there is an isomorphism $G_{\tilde{v}} \cong \mathcal{G}(v)$ taking $G_{\tilde{e}}$ onto the image of $\mathcal{G}(e)$.

One consequence of (ii) is that the natural map $\mathcal{G}(v) \rightarrow \pi_{1}(\mathcal{G})$ is injective (since it is isomorphic to the inclusion $G_{\tilde{v}} \subset G$ ). In the language of [H1] this means that $\mathcal{G}$ is developable. The tree $T$ is called the universal cover of $\mathcal{G}$. It is unique up to $G$-isomorphism. The other feature of a graph of groups is that this universal cover is not only simply connected, it is contractible.

An important application of this theory is to the problem of gluing together various $K(\pi, 1)$ spaces and then being able to decide if the result is also aspherical.

Definition 14.2. An aspherical realization of a graph of groups $\mathcal{G}$ is a $C W$ complex $B$ and a map $p: B \rightarrow \Omega^{\prime}$ so that for each vertex $v$ of $\Omega, p^{-1}(\operatorname{Star}(v))$ is a $K(\mathcal{G}(v), 1)$. Here "Star" refers to the open star of a vertex in the barycentric subdivision $\Omega^{\prime}$. (Actually, this is only an approximation of the correct definition which can be found 
Remark 14.3. It is proved in [H2], in the more general context of complexes of groups, that aspherical realizations exist and are unique up to homotopy.

We will usually denote an aspherical realization of $\mathcal{G}$ by $B \mathcal{G}$ and call it the classifying space of $\mathcal{G}$. As a definition of $\pi_{1}(\mathcal{G})$ we could take the usual fundamental group of $B \mathcal{G}$.

The following result is classical; its proof goes back to J. H. C. Whitehead.

Theorem 14.4. Let $\mathcal{G}$ be a graph of groups, $B \mathcal{G}$ an aspherical realization, and $G=\pi_{1}(\mathcal{G})$. Then $B \mathcal{G}$ is a $K(G, 1)$.

Proof. Let $E G$ be the universal cover of $K(G, 1)$. Consider the diagonal $G$-action on $E G \times T$. Projection on the second factor $E G \times T \rightarrow T$ induces a map of quotient spaces $E G \times{ }_{G} T \rightarrow \Omega$ which is clearly an aspherical realization. Hence, we can take $B \mathcal{G}=E G \times_{G} T$. On the other hand, the universal cover of $E G \times_{G} T$ is $E G \times T$ which is contractible.

Complexes of groups. Here we present a simplified version of the theory developed in [H1] and [H2]. (For the applications we have in mind we do not need the most general version of the theory.)

Definition 14.5. Let $\mathcal{P}$ be a poset. A simple complex of groups over $\mathcal{P}$ is a functor $\mathcal{G}$ from $\mathcal{P}$ to the category of groups and monomorphisms.

Remark. In the general situation of [H2], $\mathcal{P}$ need not be a poset but only a "category without loop". More importantly, $\mathcal{G}$ need not be a functor. The appropriate triangular diagrams relating compositions of morphisms need not commute on the nose but only up to conjugation by some elements in the target group; furthermore, these elements must be kept track of.

The concept of an "aspherical realization" is defined as before. Such an aspherical realization is devoted by $B \mathcal{G}$ and called the classifying space of $\mathcal{G}$. By definition, $\pi_{1}(\mathcal{G})=\pi_{1}(B \mathcal{G})$. It can also be defined via generators and relations [H2, §12.8]. A complex of groups need not be developable. Moreover, even if it is developable its universal cover need not be contractible.

Example 14.6. Suppose $\mathcal{P}=\mathcal{P}\left(\Delta^{2}\right)^{o p}$ where $\Delta^{2}$ is a 2-simplex. Define a complex of groups $\mathcal{G}$ by the following picture.

Figure 9

where $D_{m}$ denotes the dihedral group of order $2 m$. Then $\pi_{1}(\mathcal{G})$ is a Coxeter group $W$ on three generators. $\mathcal{G}$ is developable. Assume that $(1 / \pi)+(1 / q)+(1 / r)>1$. 
the Coxeter complex) and $B \mathcal{G}=E W \times{ }_{W} S^{2}$ which is not a $K(W, 1)$ (its universal cover is homotopy equivalent to $S^{2}$ ).

What is missing in higher dimensions is a hypothesis of nonpositive curvature (which is automatic in the case of a graph of groups). The proof of the following basic result is outlined by Haefliger in [H1] and some of the details are supplied in the thesis of B. Spieler [Sp].

Theorem 14.7. (Haefliger, Spieler) Let $\mathcal{G}$ be a complex of groups. Suppose that $\mathcal{G}$ admits "a metric with $K \leq 0$ ". Then

(i) $\mathcal{G}$ is developable,

(ii) its universal cover is $C A T(0)$ (and hence, contractible),

(iii) $B \mathcal{G}$ is a $K(G, 1)$ space.

Remark. Suppose $\mathcal{G}$ is a complex of groups over a poset $\mathcal{P}$. The hypothesis of nonpositive curvature means that the geometric realization of $\mathcal{P}^{\prime}$ admits a $P E$ "orbihedral structure" with $K \leq 0$. In other words, the local models of a universal cover must be $P E$ and $C A T(0)$.

Example 14.8. Suppose $(W, S)$ is a Coxeter system and that $\mathcal{S}^{f}$ is the poset defined in $\S 7$. Define a simple complex of groups $\mathcal{W}$ over $\mathcal{S}^{f}$ to be the functor $\mathcal{W}(T)=W_{T}$. Then it is easily seen that $\mathcal{W}$ is developable and that $\pi_{1}(\mathcal{W})=W$. Moreover, its universal cover is just the geometric realization of the poset $W \mathcal{S}^{f}$, i.e., it is $\Sigma^{\prime}$ (the barycentric subdivision of $\Sigma$ ). Moussong's Theorem 7.8 shows that the natural $P E$ structure on $\Sigma^{\prime}$ is $C A T(0)$, i.e., we are in the situation of Theorem 14.7 .

Example 14.9. Let $A$ be the Artin group associated to $(W, S)$. Let $\mathcal{S}^{f}$ be as above. Define a simple complex of groups $\mathcal{A}$ over $\mathcal{S}^{f}$ to be the functor $\mathcal{A}(T)=A_{T}$, where $A_{T}$ is the Artin group corresponding to $\left(W_{T}, T\right)\left(A_{T}\right.$ is an Artin group of "finite type"). It follows easily that $\pi_{1}(\mathcal{A})=A$. Moreover, it can be shown that for each $T \in \mathcal{S}^{f}, A_{T} \rightarrow A$ is injective, i.e., $\mathcal{A}$ is developable.

Consider the set,

$$
A \mathcal{S}^{f}=\coprod_{T \in \mathcal{S}^{f}} A / A_{T}
$$

partially ordered by inclusion. The geometric realization $\Phi$ of $A \mathcal{S}^{f}$ will be called the modified Deligne complex. It will be our principal object of interest in the remaining two sections. As is in the case of $\Sigma^{\prime}$, it is easy to see that $\Phi$ is simply connected and therefore, that it is the universal cover of $\mathcal{A}$.

\section{$\S 15$. Reinterpretation of the Main Conjecture.}

Recall that

$$
Y=(\Sigma \times \Sigma)-\bigcup \Sigma_{r} \times \Sigma_{r}
$$

Let $X$ denote the geometric realization of $\mathcal{S}^{f}$. Let $\pi: Y / W \rightarrow \Sigma^{\prime} / W=X$ be the map induced by projection on the first factor.

Proposition 15.1. ([CD6]). $\pi: Y / W \rightarrow X$ is an aspherical realization of $\mathcal{A}$ (from 
Proof. Basically, this is just what Deligne's Theorem (Theorem 12.2) tells us. Indeed, if $T$ is a vertex of $X$, then

$$
\pi^{-1}(\operatorname{Star}(T))=\left[(\operatorname{Star}(1, T) \times \Sigma-\bigcup \text { hyperplanes }] / W_{T}\right.
$$

which is homotopy equivalent the orbit space of the hyperplane complement for the finite Coxeter group $W_{T}$. By Deligne's result, this is a $K\left(A_{T}, 1\right)$. Thus, in the general situation, $Y / W$ is homotopy equivalent to $B \mathcal{A}$.

Corollary 15.2. The Main Conjecture holds for $(W, S)$ if and only if $\Phi$ is contractible.

Proof. We are using the form of the Main Conjecture in 12.13. By Proposition $15.1, Y / W$ is homotopy equivalent to $E A \times{ }_{A} \Phi$. Therefore, the universal cover of $Y / W$ is contractible if and only if $\Phi$ is contractible.

$\Phi$ is "building-like". As explained in $\S 12$, there is a natural epimorphism $p$ : $A \rightarrow W$. We can define a section $\varphi: W \rightarrow A$ of $p$ as follows. Given $w$ in $W$, write $w=s_{1} \cdots s_{m}$ where $s_{1} \cdots s_{m}$ is a word of minimum length for $w$. Set $\varphi(w)=x_{s_{1}} \cdots x_{s_{m}} \in A$. It can be shown that $\varphi$ is well-defined (i.e., the value of $\varphi(w)$ does not depend on the choice of minimal word). Of course, $\varphi$ is not a homomorphism. The map $\varphi$ induces a embedding of posets $W \mathcal{S}^{f} \rightarrow A \mathcal{S}^{f}$ and therefore, a simplicial embedding $\Sigma^{\prime} \rightarrow \Phi$. The translates of $\Sigma^{\prime}$ by elements of $A$ are the apartment-like subcomplexes. We have that

$$
\Phi=\bigcup_{a \in A} a \Sigma^{\prime}
$$

and in this sense, $\Phi$ is "building-like". $\Phi$ is not in fact a building: two points need not lie in a common apartment.

Nevertheless, there is an obvious idea for trying to prove $\Phi$ is contractible. Give $\Phi$ a $P E$ structure by declaring each apartment-like complex to be isometric to $\Sigma^{\prime}$ with its natural $P E$ structure (described in $\S 8$ and $\S 9$ ). Then prove $\Phi$ is $C A T(0)$. To attack this we must study links of vertices in $\Phi$.

The simplicial complexes $\hat{\Sigma}$ and $\hat{\Phi}$. Suppose for the moment that $W$ is a finite Coxeter group. Let $\Delta$ be a simplex, the codimension-one faces of which are indexed by $S$ : if $s \in S$, then $\Delta_{s}$ denotes the corresponding face. Given $x \in \Delta$, put $S(x)=\left\{s \in S \mid x \in \Delta_{s}\right\}$. Define

$$
\hat{\Sigma}_{W}=(W \times \Delta) / \sim
$$

where the equivalence relation $\sim$ is defined by $(w, x) \sim\left(w^{\prime}, x^{\prime}\right)$ if and only if $x=x^{\prime}$ and $w^{-1} w^{\prime} \in W_{S(x)} . \hat{\Sigma}_{W}$ is the usual Coxeter complex of $W$. Its poset of simplices is

$$
\left(\coprod_{T \neq S} W / W_{T}\right)^{o p}
$$

It can be identified with the triangulation of the unit sphere in $\mathbb{R}^{n}$ where the $(n-1)$ simplices are the intersection of $S^{n-1}$ with the translates of a fundamental simplicial 
angle along $\Delta_{s} \cap \Delta_{s^{\prime}}$ is $\pi / m_{s s^{\prime}}$, then the induced $P S$ structure on $\hat{\Sigma}_{W}$ is that of a round sphere.

Similarly, define

$$
\hat{\Phi}_{W}=(A \times \Delta) / \sim
$$

where $\sim$ is defined by $(a, x) \sim\left(a^{\prime}, x^{\prime}\right)$ if and only if $x=x^{\prime}$ and $a^{-1} a^{\prime} \in A_{S(x)}$. The simplicial complex $\hat{\Phi}_{W}$ is called the Deligne complex of $(W, S)$. Deligne proved, in [De], that $\hat{\Phi}_{W}$ is homotopy equivalent to a wedge of $(n-1)$-spheres (where $n-1=\operatorname{dim} \Delta$ ).

As in the previous subsection,

$$
\Phi_{W}=\bigcup_{a \in A} a \hat{\Sigma}_{W}
$$

So, $\hat{\Phi}_{W}$ is spherical-building-like. If we identify $\Delta$ with a spherical simplex as above, then each apartment-like subcomplex $a \hat{\Sigma}_{W}$ is isometric to a round sphere.

Links of vertices in $\Phi$. The vertices of $\Phi$ correspond to elements of $A \mathcal{S}^{f}$, i.e., to cosets of the form $a A_{T}, T \in \mathcal{S}^{f}$. We classify these into three types.

Type 1. $T=\emptyset$. In this case $L k(v, \Phi)=N(W, S)$ (the same link as for a vertex of $\Sigma)$.

Type 2. $T$ is maximal in $\mathcal{S}^{f}$. In this case, $\operatorname{Lk}(v, \Phi)=\hat{\Phi}_{W_{T}}$. (In the analogous case for $\Sigma^{\prime}$ the link would be a round sphere.)

Type 3. In the general case, $\operatorname{Lk}(v, \Phi)$ is the orthogonal join of a link of a simplex in $N(W, S)$ and a link of type 2 .

Thus, if we give $\Phi$ its natural $P E$ structure every link is of the form $N(W, S)$ (which is $C A T(1)$ by Moussong's Lemma and Corollary 9.4), or a Deligne complex associated to a finite Coxeter group, or a join of these two types. Thus, $\Phi$ will be $C A T(0)$ provided the following holds.

Conjecture 15.3. Let $W$ be a finite Coxeter group. Then the Deligne complex $\hat{\Phi}_{W}$ with its round metric is $C A T(1)$.

Theorem 15.4. Conjecture 15.3 implies the Main Conjecture 12.10.

It follows from a lemma of [AS, p.210] that Conjecture 15.3 holds when $W=D_{m}$, the dihedral group of order $2 m$. (The lemma asserts that $\hat{\Phi}_{D_{m}}$ has no circuits of length $\leq 2 m)$. This yields the following.

Corollary 15.5. ([CD6]) The Main Conjecture holds whenever $\operatorname{dim} \Phi=2$.

\section{$\S 16$. A cubical structure on $\Phi$.}

A Coxeter cell of type $W_{T}$ can be subdivided into combinatorial cubes, so that cubes containing the barycenter of the cell correspond to the simplices of $\hat{\Sigma}_{W_{T}}$. 
Figure 10

Given an arbitrary Coxeter system $(W, S)$, we could give $\Sigma^{\prime}(W, S)$ a cubical structure by declaring each such combinatorial cube to be a regular Euclidean cube. As in the previous section, there are three types of vertices to consider. For those of Type 2 (where $T$ is maximal), $L k\left(v, \Sigma^{\prime}\right)$ is $\hat{\Sigma}_{W_{T}}$ with its all right $P S$ structure. By [Br2, p. 29], the Coxeter complex of a finite Coxeter group is a flag complex; hence, by Gromov's Lemma, the all right structure on $\hat{\Sigma}_{W_{T}}$ is $C A T(1)$. Consider a vertex of Type 1 (where $T=\emptyset$ ). The link is $N(W, S)$ with an all right $P S$ structure. Hence, this link is $C A T(1)$ if and only if $N(W, S)$ is a flag complex. Since the links of Type 3 are orthogonal joins of versions of Type 1 and 2, we see that the cubical structure on $\Sigma$ is $C A T(0)$ if and only if $N(W, S)$ is a flag complex.

In exactly the same way, we can put a cubical structure on $\Phi$. In the case of vertices of Type 2, we have the following key lemma of [CD5, Lemma 4.3.2].

Lemma 16.1. Let $W$ be a finite Coxeter group. Then the Deligne complex $\hat{\Phi}_{W}$ is a flag complex.

For vertices of Type 1 the link is again $N(W, S)$. The conclusion is that the cubical structure on $\Phi$ is $C A T(0)$ exactly when the cubical structure on $\Sigma$ is $C A T(0)$.

Theorem 16.2. ([CD6]) The Main Conjecture is true when $N(W, S)$ is a flag complex.

Remark 16.3. Taken together, Corollary 15.5 and Theorem 16.2 constitute a proof of the Main Conjecture in most cases.

Remark 16.4. In [L], Lanner showed that $N\left(W_{T}, T\right)$ is the boundary of a simplex (an "empty simplex" in $N(W, S)$ ) if and only if $W_{T}$ is a reflection group on either hyperbolic space or Euclidean space with fundamental chamber a simplex. (See Example 11.4.) Hence, $N(W, S)$ is a flag complex if and only if for all subsets $T$ of $S$, with $\operatorname{Card}(T) \geq 3$, neither of the following conditions hold:

a) $W_{T}$ is an irreducible, affine Euclidean reflection group,

b) $W_{T}$ is a hyperbolic reflection group with fundamental chamber a simplex. 
MICHAEL W. DAVIS

\section{REFERENCES}

[ABN] A. D. Aleksandrov, V. N. Berestovskii and I. Nikolaev, Generalized Riemannian spaces, Russian Math. Surveys 41 (1986), 1-54.

[ADG] F. Ancel, M. Davis and C. Guilbault, CAT(0) reflection manifolds, in "Geometric Topology (Athens, GA, 1993)", AMS/IP Stud.Adv. Math.2.1, Amer. Math. Soc., Providence, RI, 1997, pp. 441-445..

[AG] F. Ancel and C. Guilbault, Interiors of compact contractible n-manifolds are hyperbolic $(n \geq 5)$, J. Differential Geometry 45 (1997), 1-32.

[A] E. M. Andreev, On convex polyhedra in Lobacevskii spaces, Math. USSR Sbornik 10 (1970), 413-440.

[AS] K. I. Appel and P. E. Schupp, Artin groups and infinite Coxeter groups, Invent. Math. 72 (1983), 201-220.

[BGS] W. Ballman, M. Gromov and V. Schroeder, Manifolds of nonpositive curvature, Progress in Math 61 (1985), Birkhauser, Stuttgart.

[Be] N. Benakli, Polyedres hyperboliques passage du local au global, thèse, Université de ParisSud, 1992.

[Ber] V. N. Berestovskii, Borsuk's problem on the metrization of a polyhedron, Soviet Math. Dokl. 27 (1983), 56-59.

[Bes1] M. Bestvina, The virtual cohomological dimension of Coxeter groups, in "Geometric Group Theory I", edited by G. Niblo and M. Roller, Cambridge University Press, Cambridge, 1993, 19-23.

[Bes2] _ The local homology properties of boundaries of groups, Michigan Math. J. 43 (1996), 123-129.

[BB] M. Bestvina and N. Brady, Morse theory and finiteness properties of groups, Invent. Math. 129 (1997), 445-470.

[BM] M. Bestvina and G. Mess, The boundary of negatively curved groups, J. Amer. Math. Soc. 4 (1991), 469-481.

[B] N. Bourbaki, Groupes et Algebrès de Lie, Chapters IV-VI, Masson, Paris, 1981.

[Bri] M. R. Bridson, Geodesics and curature in metric simplicial complexes, in "Group Theory from a Geometrical Viewpoint", edited by E. Ghys, A. Haefliger and A. Verjovsky, World Scientific, Singapore, 1991, 373-463.

[BH] M. Bridson and A. Haefliger, Metric Spaces of Nonpositive Curvature, Springer-Verlag, New York, 1999.

[Br1] K. Brown, Cohomology of Groups, Springer-Verlag, New York, 1982.

[Br2] - Buildings, Springer-Verlag, New York, 1989.

[Bro] M. Brown, Some applications of an approximation theorem for inverse limits, Proc. Amer. Math. Soc. 11 (1960), 478-481.

[Ca] J. W. Cannon, Shrinking cell-like decompositions of manifolds: codimension three, Ann. of Math. 110 (1979), 83-112.

[CD1] R. Charney and M. W. Davis, Singular metrics of nonpositive curvature on branched covers of Riemannian manifolds, Amer. J. Math. 115 (1993), 929-1009.

[CD2] - Strict hyperbolization, Topology 34 (1995), 329-350.

[CD3] - The Euler characteristic of a nonpositively curved, piecewise Euclidean manifold, Pac. J. Math 171 (1995), 117-137.

[CD4] - The polar dual of a convex polyhedral set in hyperbolic space, Mich. Math. J. 42 (1995), 479-510.

[CD5] _ The K( $\pi, 1)$-problem for hyperplane complements associated to infinite reflection groups, J. Amer. Math. Soc. 8 (1995), 597-627.

[CD6] L Finite $K(\pi, 1)$ 's for Artin groups, in "Prospects in Topology", edited by F. Quinn, Annals of Math. Studies 138, Princeton Univ. Press, Princeton, 1995, 110-124.

[CDM] R. Charney, M. Davis and G. Moussong, Nonpositively curved, piecewise Euclidean structures on hyperbolic manifolds, Mich. Math. J. 44 (1997), 201-208.

[CMS] J. Cheeger, W. Müller and R. Schrader, On the curvature of piecewise flat spaces, Commun. Math. Phys. 92 (1984), 405-454.

[C] S. S. Chern, On curvature and characteristic classes of a Riemannian manifold, Abh. Math. Sem. Univ. Hamburg, 20 (1956), 117-126.

[D1] M. W. Davis, Groups generated by reflections and aspherical manifolds not covered by 
[D2] _ Some aspherical manifolds, Duke Math. J. 55 (1987), 105-139.

[D3] _ Coxeter groups and aspherical manifolds, "Algebraic Topology Aarhus 1982", Lecture Notes in Math 1051, Springer-Verlag, New York, 1984, 197-221.

[D4] , Buildings are CAT(0), in "Geometry and Cohomology in Group Theory" (eds. P.Kropholler, G. Niblo, R. Stohr), London Maths. Soc. Lecture Notes 252, Cambridge Univ. Press, Cambridge, 1998, pp. 108-123.

[D5] The cohomology of a Coxeter group with group ring coefficients, Duke Math. J., 91 (1998), 297-314.

[DH] M. W. Davis and J.-C. Hausmann, Aspherical manifolds without smooth or PL structure, "Algebraic Topology", Lecture Notes in Math 1370, Springer-Verlag, New York, 1989, 135-142.

[DJ] M. W. Davis and T. Januszkiewicz, Hyperbolization of polyhedra, J. Differential Geometry 34 (1991), 347-388.

[De] P Deligne, Les immeubles de groupes de tresses généralisés, Invent. Math 17 (1972), 273-302.

[DL] W. Dicks and I. J. Leary, On subgroups of Coxeter groups, "Geometry and Cohomology in Group Theory" (eds. P.Kropholler, G. Niblo, R. Stohr), London Maths. Soc. Lecture Notes 252, Cambridge Univ. Press, Cambridge, 1998, 124-160..

[Dr] A. D. Dranishnikov, The virtual cohomological dimension of Coxeter groups, Proc. Amer. Math. Soc. 125 (1997), 1885-1891.

[E] R. D. Edwards, The topology of manifolds and cell-like maps, Proc. ICM Helsinki, 1978, 111-127.

[EG] S. Eilenberg and Ganea, On the Lusternik-Schnirelman category of abstract groups, Ann. of Math. 65 (1957), 517-518.

[FN] R. H. Fox and L. Neuwirth, The braid groups, Math. Scand. 10 (1962), 119-126.

[F] M. Freedman, The topology of four-dimensional manifolds, J. Differential Geometry 17 (1982), 357-453.

[Ge] R. Geroch, Positive sectional curvature does not imply positive Gauss-Bonnet integrand, Proc. Amer. Math. Soc., 54 (1976), 267-270.

[GH] E. Ghys and P. de la Harpe, (eds.), Sur les Groupes Hyperboliques d'apres Mikhael Gromov, Prog. Math. 83, Birkhauser, Boston,1990.

[G] M. Gromov, Hyperbolic groups, in "Essays in Group Theory", edited by S. M. Gersten, M.S.R.I. Publ. 8, Springer-Verlag, New York, 1987, 75-264.

[H1] A. Haefliger, Complexes of groups and oribhedra, in "Group Theory from a Geometrical Viewpoint", edited by E. Ghys, A. Haefliger and A. Verjousky, World Scientific Singapore, 1991, 504-540.

[H2] _ Extensions of complexes of groups, Ann. Inst. Fourier, Grenoble 42, 1-2 (1992), 275-311.

[K] M. A. Kervaire, Smooth homology spheres and their fundamental groups, Amer. Math. Soc. Trans. 144 (1969), 67-72.

[L] F. Lanner, On complexes with transitive groups of automorphisms, Comm. Sem. Math. Univ. Lund 11 (1950), 1-71.

[Lek] H. van der Lek, The homotopy type of complex hyperplane complements, Ph.D. thesis, University of Nijmegan, 1983.

[M] G. Moussong, Hyperbolic Coxeter groups, Ph.D. thesis, The Ohio State University, 1988.

$[\mathrm{RH}] \quad$ I. Rivin and C. Hodgson, A characterization of compact convex poyhedra in hyperbolic 3-space, Invent. Math. 111 (1993), 77-111.

[Ro] D. Rolfsen, Strongly convex metrics in cells, Bull. Amer. Math. Soc. 78 (1968), 171-175.

[R] M. Ronan, Lectures on Buildings, Academic Press, San Diego, 1989.

[S] M. Salvetti, Topology of the complement of real hyperplanes in $\mathbb{C}^{n}$, Invent. Math. 88 (1988), 603-618.

[Se] J. P. Serre, Trees, Springer-Verlag, New York, 1989.

[Sp] B. Spieler, Nonpositively curved orbihedra, Ph.D. thesis, The Ohio State University, 1992.

[Sta1] J. R. Stallings, The piecewise-linear structure of Euclidean space, Proc. Cambridge Phil. Soc. 58 (1962), 481-488.

[Sta2] _ On torsion-free groups with infinitely many ends, Ann. of Math. 88 (1968), 312-334. 
[Sto] D. Stone, Geodesics in piecewise linear manifolds, Trans. Amer. Math. Soc. 215 (1976), 1-44.

[Thp] P. Thurston, The topology of 4-dimensional G-spaces and a study of 4-manifolds of nonpositive curvature, Ph.D. thesis, University of Tennessee, 1993.

[Th] W. Thurston, The geometry and topology of 3-manifolds, reproduced lecture notes (1979), Princeton University.

[T] C. Tomei, The topology of the isospectral manifold of tridiagonal matrices, Duke Math. J. 51 (1984), 981-996.

[V] E. B. Vinberg, Discrete linear groups generated by reflections, Math. USSR Izvestija 5, No. 5 (1971), 1083-1119.

[W] D. W. Walkup, The lower bound conjecture for 3- and 4-manifolds, Acta Math., 125 (1970), 75-107.

[Wr] D. Wright, Contractible open manifolds which are not covering spaces, Topology 31 (1992), 281-291.

Department of Mathematics, The Ohio State University, $231 \mathrm{~W}$. 18th Avenue, Columbus, Ohio 43210

MDAVIS@MATH.OHIO-STATE.EDU 